\title{
Quantitative analysis of nuclear pore complex organization in Schizosaccharomyces pombe
}

\author{
Joseph M. Varberg ${ }^{1, 凶}$, Jay R. Unruh ${ }^{1}$, Andrew J. Bestul ${ }^{1}$, Azqa A. Khan ${ }^{1}$, and Sue L. Jaspersen ${ }^{1,2, 凶}$ \\ ${ }^{1}$ Stowers Institute for Medical Research, Kansas City, MO, United States \\ ${ }^{2}$ Department of Molecular and Integrative Physiology, University of Kansas Medical Center, Kansas City, KS, United States
}

\begin{abstract}
The number, distribution and composition of nuclear pore complexes (NPCs) in the nuclear envelope (NE) varies between cell types and changes during cellular differentiation and in disease. To understand how NPC density and organization is controlled, we analyzed NPC number and distribution in the fission yeast Schizosaccharomyces pombe using structured illumination microscopy. The small size of yeast nuclei, genetic features of fungi and our robust image analysis pipeline allowed us to study NPCs in intact nuclei under multiple conditions. Our data revealed that NPC density is maintained across a wide range of nuclear sizes. Regions of reduced NPC density are observed over the nucleolus and surrounding the spindle pole body (SPB). Lem2mediated tethering of the centromeres to the SPB is required to maintain NPC exclusion, which is important for timely mitotic progression. These findings provide a quantitative understanding of NPC number and distribution in S. pombe and show that interactions between the centromere and the $\mathrm{NE}$ influences local NPC distribution.
\end{abstract}

nuclear pore complex | nuclear envelope | Lem2 | nucleolus | spindle pole body

Correspondence: jvarberg@stowers.org, slj@stowers.org

\section{Introduction}

Nuclear pore complexes (NPCs) facilitate nucleocytoplasmic transport, organize the genome, influence gene expression and facilitate DNA repair (1-3). Each NPC is composed of multiple copies of $\approx 30$ individual nucleoporin (Nup) proteins, which are organized around a central channel in eightfold symmetry (4-7). The NPC is anchored in the nuclear envelope (NE) by transmembrane Nups and through interactions between specific Nups and lipids of the nuclear membrane. Decades of research in a variety of systems has identified conserved functions for Nups in NPC assembly and transport and has mapped their organization within the structure of the NPC at nearly atomic resolution (8-11).

In contrast to our understanding of NPC structure, the mechanisms that control NPC density, distribution and composition remain poorly understood. Early studies using electron microscopy (EM) showed that NPC density is highly variable between species and cell types (12-14). NPC density does not appear to correlate with nuclear size or DNA content, but it is associated with metabolic activity (15) perhaps explaining links between changes in NPC density and cancer $(16,17)$ or in response to external signals (18-22). The remarkably long half-lives of many Nups (23-25) has led to a proposal that the number of NPCs in a cell is likely regulated at the stage of NPC assembly (reviewed in (26)). In metazoans and in budding yeast, the number of NPCs in the NE roughly doubles during interphase nuclear growth (21, 27-30). NPC assembly during the cell cycle is positively regulated by cyclindependent kinases (Cdks) (31) and negatively regulated by phosphorylation of NPC assembly factors by extracellularregulated stress kinase (ERK) (32). However, some debate remains over the ubiquitous role of the ERK-mediated pathway for regulation of NPC density $(33,34)$.

Once assembled into the NE, NPCs adopt a variety of nonrandom distributions, ranging from pairs and clusters to higher-order linear and hexagonal arrays (12). Plant, animal and fungal nuclei have reduced NPC density in regions over the nucleolus and near sites of contact between the nucleus and cytosolic organelles, such as the vacuole/lysosome, Golgi apparatus and mitochondrion (12, 27, 35-40). Despite decades of work clearly demonstrating non-random NPC distributions in multiple cell types, little is known about how these patterns are formed and maintained. In metazoans, NPC distribution is mediated at least in part through the nuclear lamina (33, 41-43). However, as both plants and fungi lack lamins, additional factors must serve to regulate NPC distribution. LAP2-emerin-MAN1 (LEM)-domain proteins, which associate with the inner nuclear membrane (INM) throughout eukaryotes, are leading candidates; for example, emerin is enriched at pore-free regions of the $\mathrm{NE}$ in cultured cells (44). In budding yeast, NPC density is increased in the region of the NE near the spindle pole body (SPB), suggesting that either the SPB itself or associated factors control NPC recruitment or assembly $(27,39,45)$.

Analysis of NPC composition in the region over the nucleolus in Saccharomyces cerevisiae showed that nucleolarassociated NPCs lack two Nups, Mlp1 and Mlp2 (46, 47). These orthologs of vertebrate Nup Tpr (translocated promoter region) are core structural components of the nuclear basket, a nucleoplasmic extension of the NPC that serves as a binding site for chromatin, proteasomes and other factors (48-51). Despite the clear evidence that $S$. cerevisiae maintain compositionally distinct populations of NPCs in subregions of the NE, we currently lack any mechanistic insight into how this is achieved. Further, it is unknown whether this is a unique property of budding yeast nuclei, as may be the case for other aspects of fungal NPC biology including mechanisms controlling inheritance of NPCs during mitotic divisions (which rely on the $S$. cerevisiae bud-neck structure) (52-56) and NPC remodeling during budding yeast meio- 
sis (which is not seen in S. pombe) $(57,58)$. Identifying the mechanisms that control heterogeneity in NPC composition and distribution are of great interest, as transcriptomic and proteomic studies in metazoans have identified cell-type specific Nup expression patterns and shown that changes in NPC composition that are critically important for cell development, differentiation and progression of various diseases (59-64). These findings, in combination with the evidence for NPC compositional heterogeneity within individual nuclei in yeast, highlight the emerging concept that subpopulations of NPCs with distinct compositions and potentially specialized functions may exist at specific locations within the nuclear envelope (65).

Using $S$. pombe as a model system, we combined multiple quantitative imaging approaches, including threedimensional structured illumination microscopy (3D-SIM), to examine the number, distribution, and composition of NPCs in whole nuclei. We quantify NPC number under a range of conditions and show that fission yeast maintains a constant NPC density throughout its life cycle. NPC density appears to be maintained through a mechanism that links NPC assembly to available NE surface area. Experiments using 3D-SIM and live-cell imaging revealed a common structural organization of NPC clusters and identified two distinct behaviors of clusters during mitotic cell division. We show that the previously reported reduction of NPC density and alteration of NPC basket composition over the nucleolus-facing region of the NE is conserved in fission yeast. Additionally, NPCs are excluded from the NE region surrounding the SPBs by Lem 2 and other factors.

\section{Results}

3D-SIM image analysis pipeline for NPC quantitation. We developed an imaging and analysis pipeline to visualize and count NPCs in fission yeast following three-dimensional structured illumination microscopy (3D-SIM) of entire nuclei containing endogenously tagged Nups. This approach provides a roughly two-fold increase in resolution as compared to conventional light microscopy, with lateral resolution that approaches the size of the yeast nuclear pore $(\approx 90-120 \mathrm{~nm}$ diameter) $(11,66)$. Individual foci corresponding to single NPCs (FWHM $=121.6-136.5 \mathrm{~nm}, 95 \% \mathrm{CI}$ ) as well as larger foci that likely represent clusters of NPCs that cannot be fully resolved by SIM were detected throughout the nucleus (Fig. 1A). NPCs could be visualized using various tagged Nups, including representatives from each NPC subcomplex (Fig. 1B). NPC number and position, nuclear size and cell cycle stage could be extracted from images using the strategy outlined in Fig. 1C and these values could be used to derive measurements of nuclear surface area and NPC density (see Materials and Methods).

In $S$. pombe, nuclear size increases through interphase to maintain a constant nuclear-to-cell volume ratio (67). We observed that the number of NPCs also increases through interphase to maintain a constant NPC density (Table S1, Fig. 1D-E). We observed occasional differences in NPC density between mother and daughter nuclei produced by the symmetric mitotic division in $S$. pombe, reminiscent of the elevated NPC density observed for daughter nuclei produced by the asymmetric mitosis in S. cerevisiae (52). Visualization of Cdc7-GFP, a kinase that preferentially localizes to the "new" SPB during anaphase B (68), showed that the asymmetric NPC densities we occasionally observed is random with respect to the inheritance of the "old" or "new" SPB (Fig. S1A). During the late stages of $S$. pombe mitosis, a subset of NPCs localize to the membrane bridge where they facilitate active transport before being selectively disassembled to trigger localized NE breakdown and spindle disassembly (69-71). In agreement with these findings, we observed NPCs undergoing disassembly in the anaphase bridge region: NPCs contained transmembrane (Cut11) and structural nucleoporins (Nup37) but lacked the basket (Nup60) (Fig. S1B). Because of their dynamic nature, bridge NPCs were excluded from quantitative cell cycle measurements.

Despite the improved lateral resolution offered by SIM, clustering of NPCs and the comparatively reduced axial resolution likely leads to undercounting of NPCs using 3D-SIM. To determine the extent of undercounting, we applied our analysis pipeline to simulated datasets modeling a range of NPC densities (Fig. S1C). For simulated densities ranging from 2-5 $\mu^{2}$, the measured values for NPC density and NE surface area fell within $10 \%$ of the true simulated values; the percent error increased in a density-dependent manner, with values ranging from $10-30 \%$ for densities up to $10 \mathrm{NPCs} / \mu$ $\mathrm{m}^{2}$. Due to this undercounting, we used a secondary approach that did not rely on segmentation of individual NPCs from 3D-SIM images to measure NPC density. Comparison of nuclear size and total Nup-GFP intensities through the cell cycle showed similar increases in nuclear size and total NPC number, while NPC density (total Nup-GFP intensity per unit area) remained constant (Fig. S1D).

After correcting for the undercounting observed for our 3DSIM approach, our analysis estimates that the average midG2 stage fission yeast nucleus contains between 115-137 \pm 22-26 NPCs, with a nuclear surface area of $18.4 \pm 2.8 \mu \mathrm{m}^{2}$, for a density of 6.3-7.4 NPCs $/ \mu^{2}(n=174)$. These values are lower than those reported for budding yeast (ranging from 9$15 \mathrm{NPCs} / \mu^{2},(13,27,72)$ but are similar to NPC densities reported for other cell types (ranging from $4.5-8 \mathrm{NPCs} / \mu \mathrm{m}^{2}$ ) $(13,14,18,30,31)$. While similar trends in NPC number and density were observed using multiple tagged nucleoporins, variability in the number of NPCs detected was observed when comparing datasets between different tagged Nups (Fig. S1E). As a result, all experiments comparing genetic backgrounds or treatment conditions were performed in cells expressing the same tagged Nup.

NPC density is controlled in a NE surface area-dependent manner. NPC density might be controlled by a mechanism coupling NPC assembly with available NE surface area (32). To explore this possibility in $S$. pombe, we examined NPC density in cells with nuclei covering a broad range of sizes. Meiotic progeny, known as spores, have a 
bioRxiv preprint doi: https://doi.org/10.1101/2021.08.11.455278; this version posted August 11, 2021. The copyright holder for this preprint (which was not certified by peer review) is the author/funder, who has granted bioRxiv a license to display the preprint in perpetuity. It is made available under aCC-BY 4.0 International license.

A

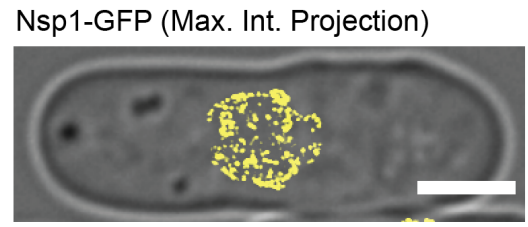

Top Slice

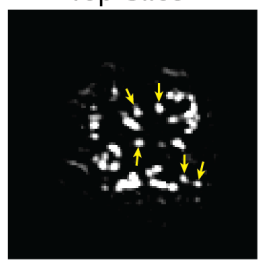

C

Nuclear segmentation

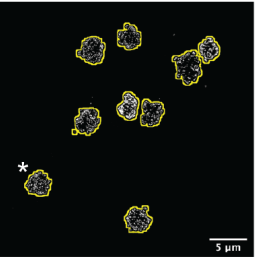

NPCs detection and position extraction from individual nuclei

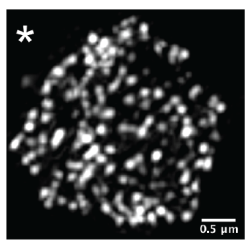

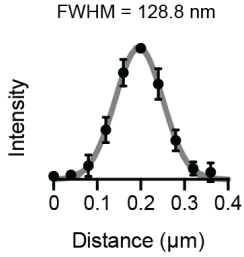

Cell length and morphology

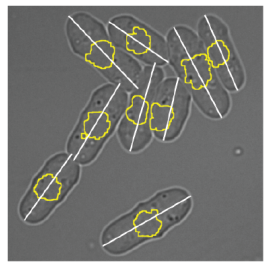

Computation of $3 \mathrm{D}$ convex hull for nuclear shape, surface area and volume

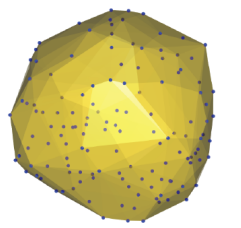

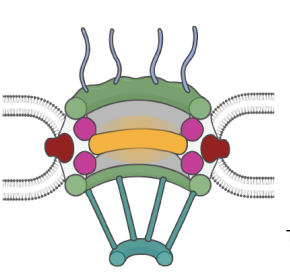
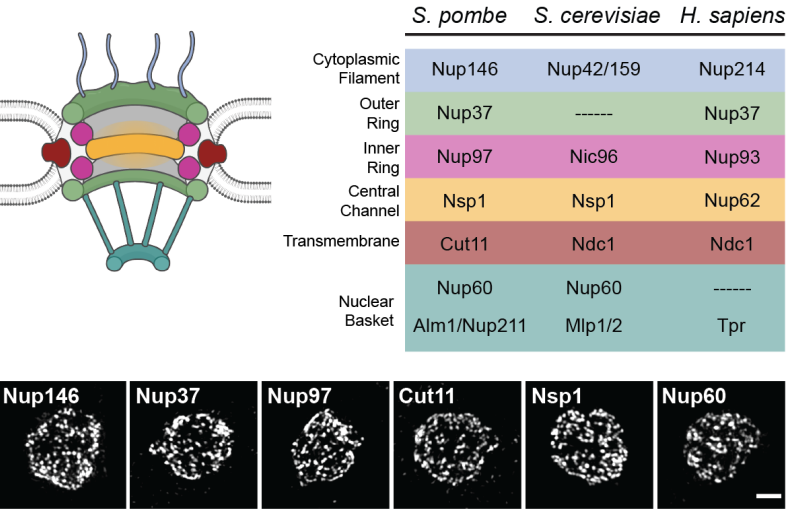

D
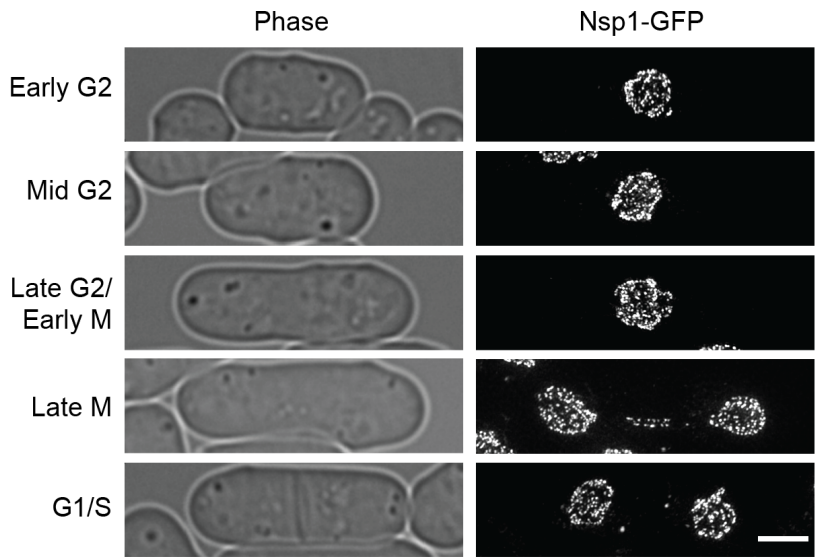

$\mathrm{E}$
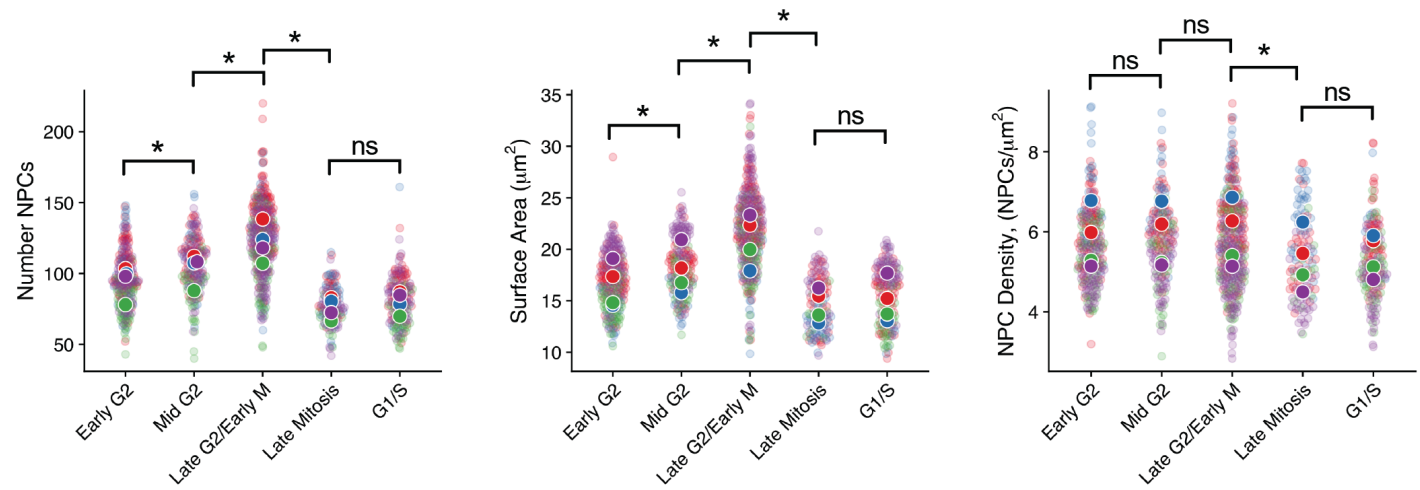

Fig. 1. A 3D-SIM imaging and analysis pipeline to measure NPC number and density in S. pombe A) 3D-SIM image of Nsp1-GFP overlayed on transmitted light image. Bar, $3 \mu \mathrm{m}$. Arrows show five foci representing single NPCs, which were fit to a Gaussian function to generate an average NPC intensity profile (FWHM, full-width half-maximum). B) NPC schematic with sub-complexes colored to match the table of individual Nups shown on right, with representative 3D-SIM images below. Bar, $1 \mu$ m. C) Pipeline for NPC analysis. D) Representative 3D-SIM image from each cell cycle stage. Bar, $3 \mu \mathrm{m}$. E) Mean number of NPCs, nuclear surface area and NPC density measurements from four independent replicates. Significant differences $\left(^{*}\right)$ determined using Wilcoxon rank sum tests. ns, not significant. 
bioRxiv preprint doi: https://doi.org/10.1101/2021.08.11.455278; this version posted August 11, 2021. The copyright holder for this preprint (which was not certified by peer review) is the author/funder, who has granted bioRxiv a license to display the preprint in perpetuity. It is made available under aCC-BY 4.0 International license.

A
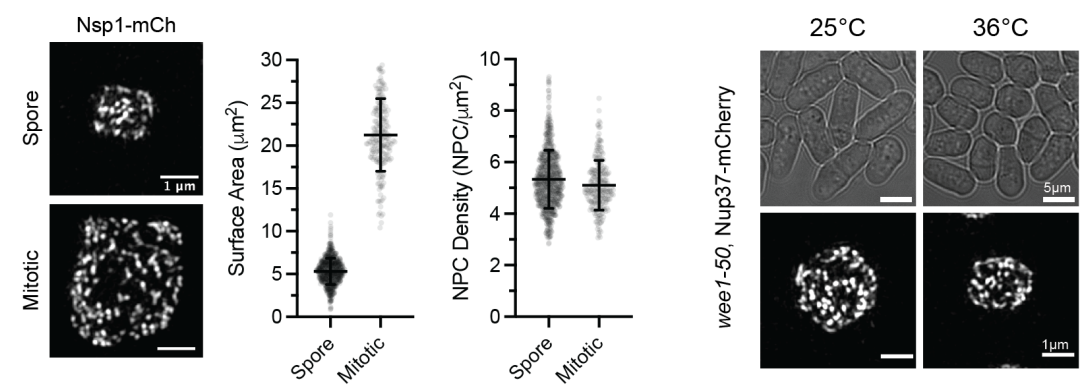
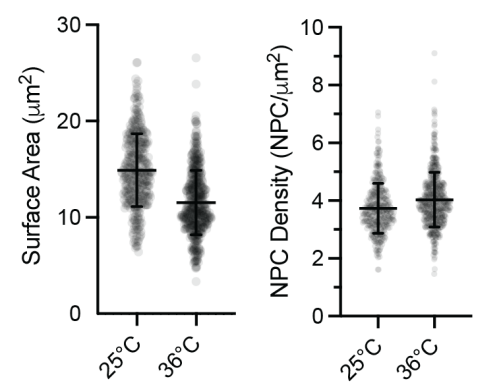

C
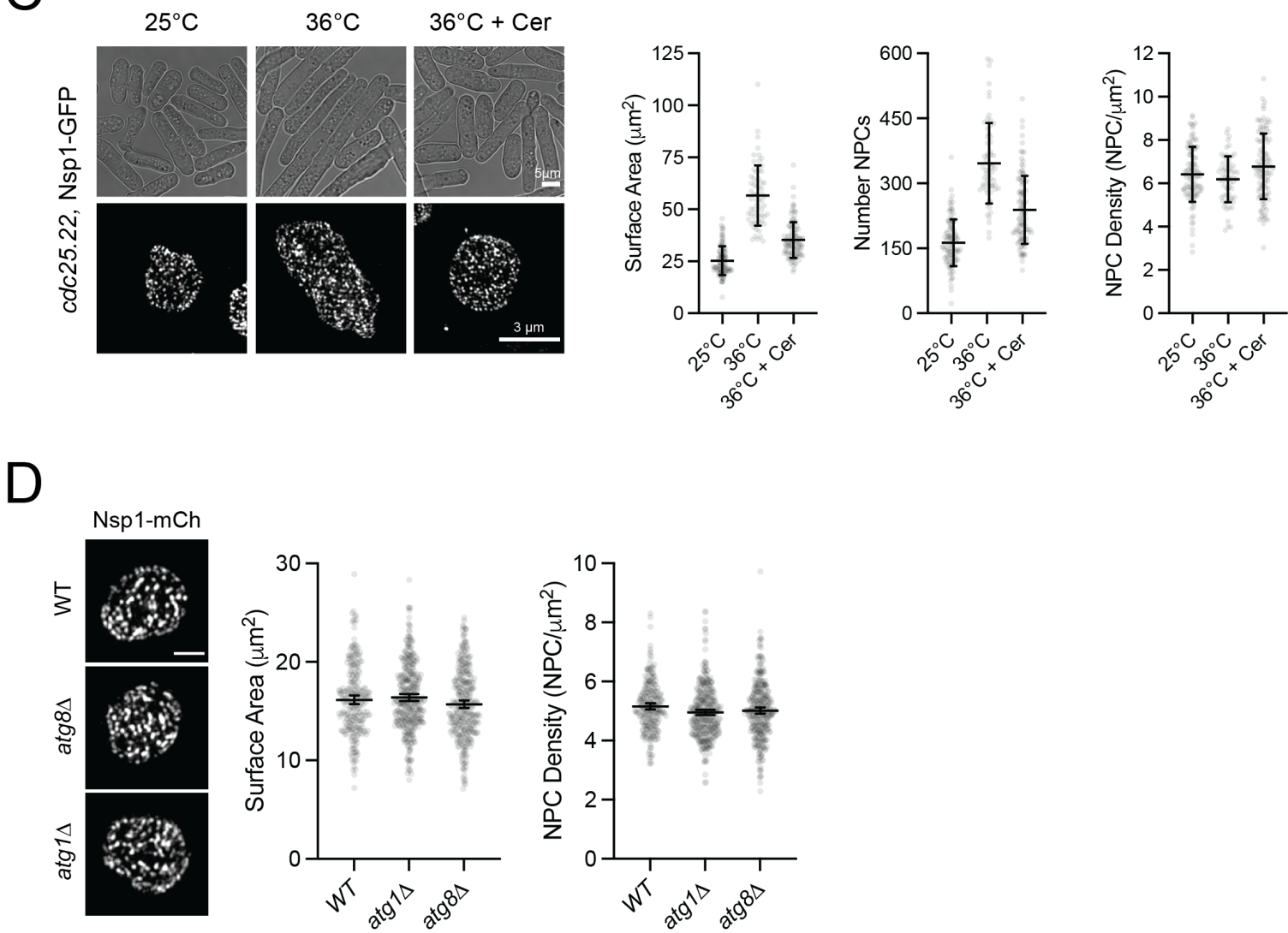

Fig. 2. Surface area-dependent maintenance of NPC density A) 3D-SIM image of Nsp1-mCh in nuclei from meiotic spores (top) or mitotic G2 (bottom) nuclei, with quantitation on right. B) $3 \mathrm{D}$-SIM and quantitation of Nup37-mCh NPCs in wee1.50 mutants grown at $25^{\circ} \mathrm{C}$ or shifted to $36^{\circ} \mathrm{C}$ for $3.5 \mathrm{~h}$. C) $3 \mathrm{D}-\mathrm{SIM}$ and quantitation of Nsp1-GFP in cdc25.22 mutants at $25^{\circ} \mathrm{C}$ or shifted to $36^{\circ} \mathrm{C}$ for $3.5 \mathrm{~h}$ in the absence or presence of $10 \mu \mathrm{M}$ Cerulenin (Cer). D) 3D-SIM and quantitation of Nsp1-mCh NPCs in wild-type, atg $8 \Delta$ and $\operatorname{atg} 1 \Delta$ cells. Bars, $1 \mu \mathrm{m}$.

similar NPC density to mitotic cells despite having nuclei with 3-4 fold lower nuclear surface area (Fig. 2A) (Nsp1$\mathrm{mCh}$ : Spores $=5.4 \pm 1.2 \mathrm{NPCs} / \mu \mathrm{m}^{2}, \mathrm{n}=718 ;$ Mitotic, $=5.1$ $\left.\pm 0.93 \mathrm{NPCs} / \mu^{2}, \mathrm{n}=207\right)$. Similarly, a constant NPC density was maintained when nuclear size was reduced in mitotic cells using a temperature-sensitive allele of Wee1 kinase (wee 1.50), a negative regulator of the cyclin-dependent kinase Cdk1/Cdc2 (73) (Fig. 2B) (Nup37-mCh: $25^{\circ} \mathrm{C}=3.7$ $\pm 0.86 \mathrm{NPCs} / \mu \mathrm{m}^{2}, \mathrm{n}=382 ; 36^{\circ} \mathrm{C}=4.0 \pm 0.95 \mathrm{NPCs} / \mu \mathrm{m}^{2}$, $\mathrm{n}=506$ ). Cells expressing a temperature-sensitive mutation in the Cdk1/Cdc2-phosphatase Cdc25 (cdc25.22) arrest at the
G2/M boundary yet continue to increase both cell and nuclear size (74). During $c d c 25.22$ arrest, both nuclear surface area and the number of NPCs roughly doubled over the 3.5-hour incubation, allowing NPC density to be maintained (Nsp1GFP: $25^{\circ} \mathrm{C}=6.4 \pm 1.3 \mathrm{NPCs} / \mu^{2}, \mathrm{n}=106 ; 36^{\circ} \mathrm{C}=6.2 \pm 1.0$ $\mathrm{NPCs} / \mu \mathrm{m}^{2}, \mathrm{n}=63$ ) (Fig. 2C). The increase in NPC number was dependent on NE membrane expansion during arrest, as chemical inhibition of fatty acid synthesis by treatment with cerulenin blocked nuclear growth while NPC density was maintained $\left(36^{\circ} \mathrm{C}+\right.$ Cerulenin $=6.8 \pm 1.5 \mathrm{NPCs} / \mu \mathrm{m}^{2}$, $\mathrm{n}=110$ ) (Fig. 2C). Yeast lacking core components of the au- 
tophagy machinery (atg8 8 or $\operatorname{atg} 1 \Delta$ ) (75) that targets NPCs for degradation during nutrient deprivation do not show increased NPC density compared to wild-type cells, suggesting that autophagy is not used to remove NPCs to maintain NPC density (Fig. 2D) (Nsp1-mCh: WT $=5.2 \pm 0.83 \mathrm{NPCs} / \mu \mathrm{m}^{2}$, $\mathrm{n}=252 ; \operatorname{atg} 1 \Delta=5.0 \pm 0.87 \mathrm{NPCs} / \mu^{2}, \mathrm{n}=359 ; \operatorname{atg} 8 \Delta=5.0$ $\pm 0.97 \mathrm{NPCs} / \mu \mathrm{m}^{2}, \mathrm{n}=326$ ). These results support a model whereby NPC density is maintained by a mechanism that restricts the assembly of new NPCs in the absence of increased available NE surface area.

NPC cluster organization and dynamics. Our ability to observe NPCs throughout entire nuclei using 3D-SIM at near single-NPC resolution allowed us to evaluate higher level NPC organization. NPC clustering is common phenotype in different cell types and in mutants defective in NPC assembly. Using 3D-SIM, we compared NPC distribution in wild-type cells to two previously described clustering mutants: nup $132 \Delta$ and nem $1 \Delta$ (76-78).

Widefield and confocal images of NPC clusters in nup $132 \Delta$ mutants often appear as one or two large clusters, however, 3D-SIM images revealed the presence of multiple smaller clusters distributed throughout the NE (Fig. 3A). The majority of nup $132 \Delta$ nuclei displayed normally distributed NPCs or very mild NPC clustering, with only $14 \%$ displaying moderate to severe clustering (Fig. 3A). Unexpectedly, we frequently observed NPC clusters organized in a ring-like structure with diameters ranging from 250-300 nm (Fig. 3B). In rare cases, ring-like NPC clusters were also observed in wildtype cells, suggesting that these are not simply a unique phenotype of nup $132+$ deletion. Clustering increased in aged nup 132 $\Delta$ cells grown on plates (Fig. 3C), consistent with previous reports (76). Similar rings were also observed in neml $\Delta$ cells, which have increased rates of lipid synthesis that alters NE morphology and NPC distribution (70, 78-80) (Fig. 3D).

To examine the dynamics of the NPC clusters through the cell cycle, we performed time-lapse imaging of nup $132 \Delta$ and neml $\Delta$ cells and monitored NPC cluster dynamics in single cells. These experiments revealed two surprisingly different behaviors for clustered NPCs. In neml $\Delta$ mutants, NPC clustering became more severe as nuclei prepared to divide. NPC clusters were frequently enriched in the anaphase bridge, along with excess membrane (Fig. 3E, S2). Following completion of nuclear division, the resulting daughter nuclei had normal NE morphologies and NPC densities equivalent to wild-type nuclei (Fig. S2). This suggests that neml $\Delta$ nuclei can remove excess NE membrane and NPCs during mitosis via the anaphase bridge. In contrast, NPC clusters in nup $132 \Delta$ nuclei coalesced into larger clusters that preferentially localized to the SPBs in mitosis (Fig. 3G). SPBassociated clusters are then segregated into the mother and daughter nuclei as cells complete mitosis. These observations show that at least two independent mechanisms exist to control NPC cluster dynamics and transmission during $S$. pombe nuclear division.
Reduced NPC density and altered basket composition over the nucleolus. We observed a clear reduction in NPC density over the nucleolus (visualized using the RNA polymerase I subunit Nuc1) (81) from middle slices of 3D-SIM images (Fig. 4A), suggesting that a similar reduction of NPC density over the nucleolus occurs in S. pombe like $S$. cerevisiae (39). To quantitatively assess NPC density over this region we compared the intensities for multiple Nups from all subcomplexes over the nucleolus with intensities over the rest of the nuclear envelope (Fig. 4B-C). This confirmed that NPC density is reduced by $\approx 20 \%$ over the nucleolus-facing region of the $\mathrm{NE}$ and showed $\mathrm{a} \approx 50 \%$ average reduction in the $S$. pombe Tpr orthologs Alm1 and Nup211 $(48,51,82)$ (Fig. 4D). Like budding yeast, the NE region over the nucleolus has reduced NPC density and contains a unique population of NPCs that specifically lack the Tpr basket nucleoporins.

NPCs are excluded from the SPB-proximal region throughout the mitotic cell cycle. In contrast to the nucleolar region, increased NPC density is found near the budding yeast SPBs (27), possibly due to a role of NPCs in NE remodeling during SPB insertion into the NE (45). EM analysis of fission yeast SPBs failed to identify an increased presence of NPCs within $\approx 200 \mathrm{~nm}$ of the SPB regardless of cell cycle stage (Fig. 5A). To examine the distribution of NPCs relative to the SPB at high resolution, we used single particle averaging of multiple 3D-SIM images (SPA-SIM); this approach has allowed us to visualize SPB-proximal proteins in budding and fission yeast (83-86). In SPA-SIM, the position of the two duplicated but unseparated SPBs are used as fiduciary points to realign images from multiple nuclei. To ensure that NPC distribution is visualized from a top-down perspective, we restricted our SPA-SIM analysis to SPBs that were centrally localized in the $\mathrm{x}-\mathrm{y}$ plane with respect to the nucleus (see Materials and Methods). A composite image was then generated representing the average distribution of proteins of interest with respect to the SPBs.

Consistent with EM analysis of NPC distribution around the fission yeast SPB, we observed a clear zone of NPC exclusion surrounding the SPBs in asynchronous populations of exponentially growing cells (Fig. 5B). This exclusion was not seen for Les1, an INM protein that is not a component of the NPC (70) or from simulations of randomly distributed NPCs (Fig. 5B). This exclusion zone was highly reproducible and cell cycle independent (Fig. 5D-E), with an average diameter of $\approx 200 \mathrm{~nm}(\mathrm{FWHM}=183.8-217.2 \mathrm{~nm}, 95 \% \mathrm{CI})$. We considered that exclusion of NPCs from the SPB could be due to forces exerted on the SPBs during interphase through the activity of microtubule-based motor proteins (87). However, the exclusion zone was not altered following disruption of microtubules by treatment with the depolymerizing agent methyl benzimidazol-2-yl carbamate (MBC) $(87,88)$ (Fig. 5C).

We envisioned at least two possible models that could explain the NPC exclusion near the SPB. In the first, NPCs could be physically excluded from this region, perhaps through the 
bioRxiv preprint doi: https://doi.org/10.1101/2021.08.11.455278; this version posted August 11, 2021. The copyright holder for this preprint (which was not certified by peer review) is the author/funder, who has granted bioRxiv a license to display the preprint in perpetuity. It is made available under aCC-BY 4.0 International license.
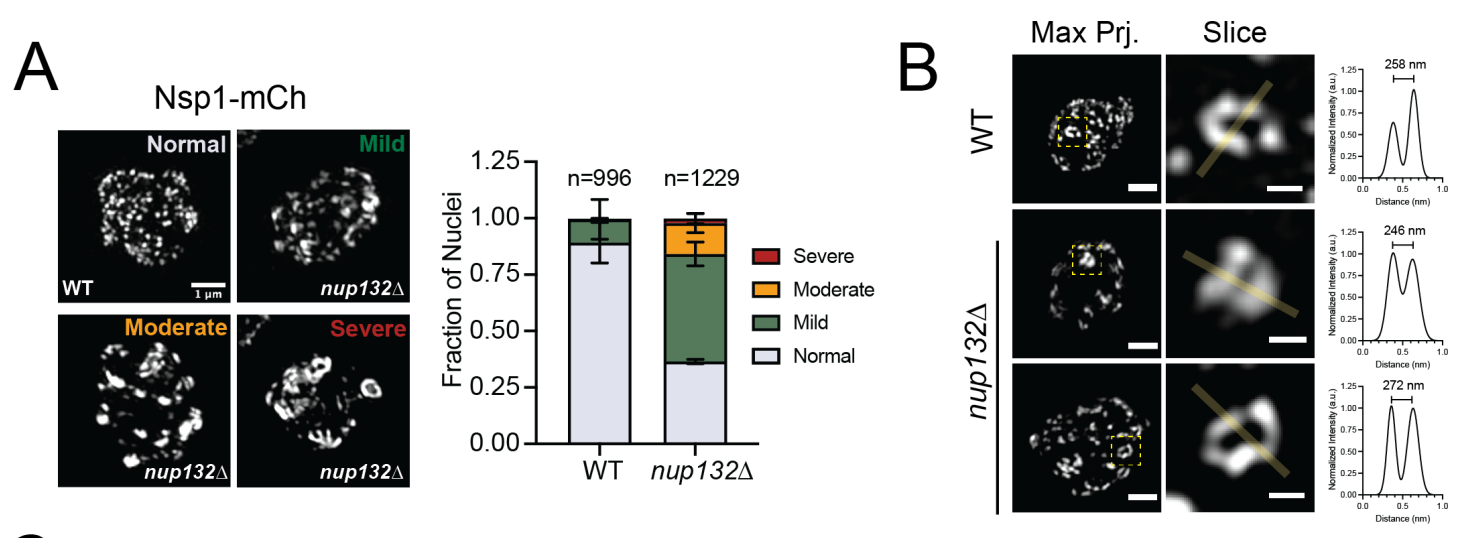

C
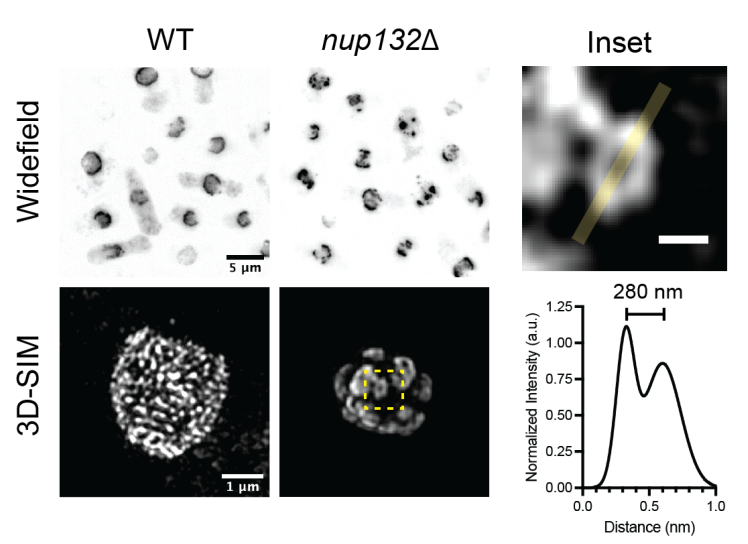

$D$
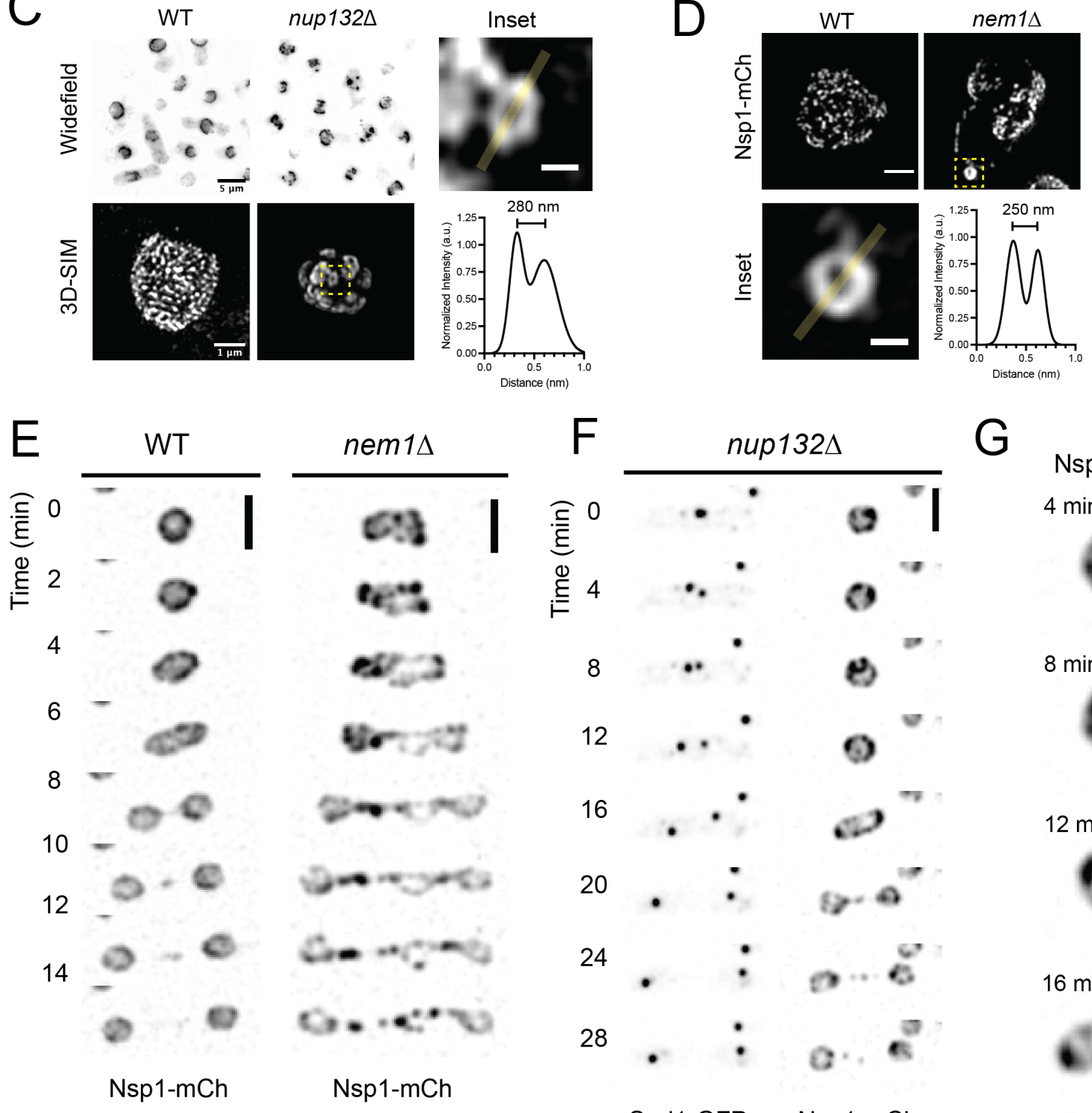

G

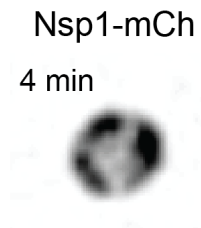

Sad1-GFP Nsp1-mCh

Fig. 3. NPC cluster organization and dynamics A) 3D-SIM of Nsp1-mCh in wild-type cells and in nup132 mutants. Quantitation of the frequency of clustering phenotypes from two independent replicates. Bar, $1 \mu \mathrm{m}$. B) Ring-like NPC clusters observed by 3D-SIM from cells in (A), including projections of the entire nucleus (left; bar, $1 \mu \mathrm{m}$ ) and magnified region (center; bar, $300 \mathrm{~nm}$ ). Line profiles were taken across the indicated regions of the magnified region, with the corresponding intensity profiles shown at right. C) Clustering increases in nup $132 \Delta$ cells grown on YES agar plates at $25^{\circ} \mathrm{C}$ for $7 \mathrm{~d}$. D) 3D-SIM of Nsp1-mCh NPCs in wild-type and nem1 $1 \Delta$ mid-G2 stage nuclei (Bar, 1

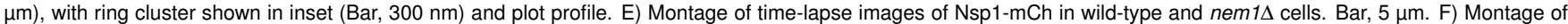
Nsp1-mCh and the SPB component, Sad1-GFP, in nup132 $\Delta$ mutants. Nsp1-mCh intensity at the SPB relative to the average NE intensity is plotted, with the mitotic timepoints shown in the montage highlighted in gray. Bar, $5 \mu \mathrm{m}$. G) Insets of nuclei at the indicated time points from montage in $\mathrm{F}$. Bar, $3 \mu \mathrm{m}$. 
A

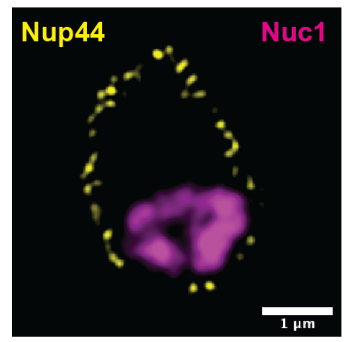

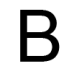

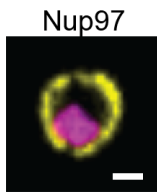

Nup60

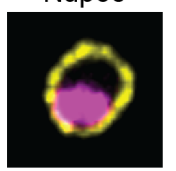

Seh1

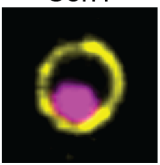

Nup124

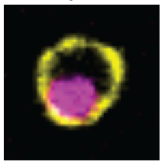

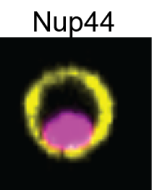

Alm1

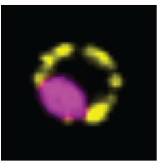

Nup146

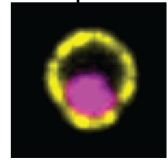

Nup211

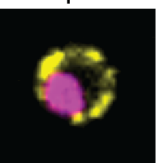

Average Normalized Intensity (a.u.)

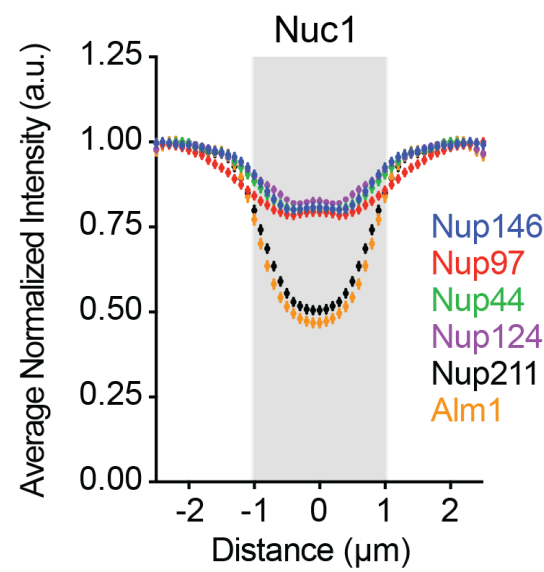

D
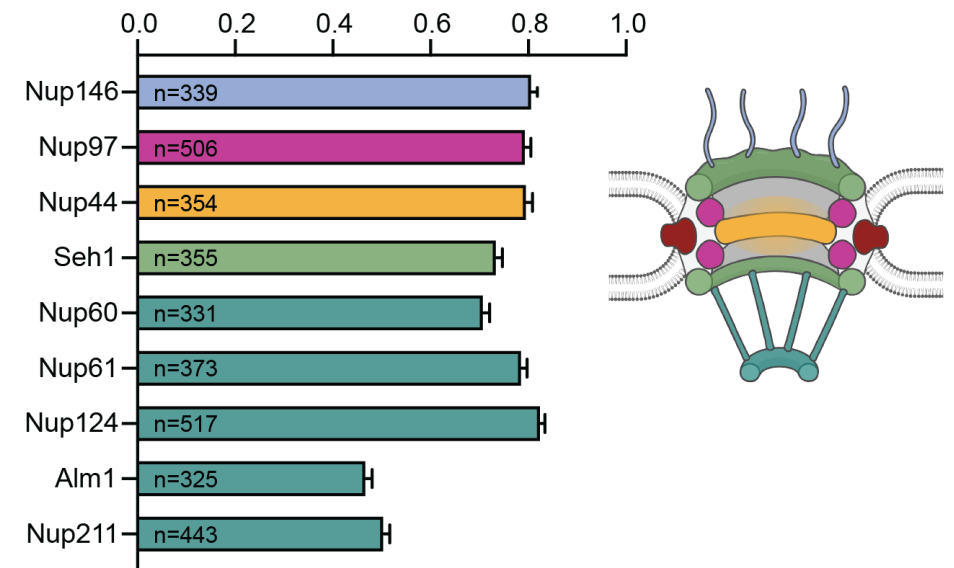

Fig. 4. Reduced NPC density and altered NPC composition at the nucleolus A) Middle slice from 3D-SIM image of Nup44-GFP NPCs and the nucleolus (Nuc1-mCh, processed with Gaussian blur). Bar, $1 \mu \mathrm{m}$. B) Confocal images of middle slices of nuclei showing Nups (yellow) and the nucleolus (magenta). Bar, $1 \mu \mathrm{m}$. C) Averaged profiles of Nup intensities at the NE relative to the nucleolus (gray, based on FWHM $\pm 95 \% \mathrm{Cl}$ of Nuc1-mCh). D) Average Nup intensity at position 0 in intensity profiles from (C), organized according to NPC subcomplex based on the schematic in Figure 1B. Error bars, SD. N, number of nuclei analyzed for panels C and D.

presence of nuclear membrane proteins localized to the SPB region. This could include factors such as the SUN (Sad1Unc-84 homology) domain-containing protein Sad1, which interacts with KASH (Klarsicht, ANC-1, Syne Homology) domain proteins $\mathrm{Kms} 1$ and $\mathrm{Kms} 2$ to form a LINC (Linker of Nucleoskeleton and Cytoskeleton) complex that tethers the cytosolic SPB to the NE $(89,90)$, or through proteins that tether centromeres to the NE (reviewed in (91)). Alternatively, the exclusion could represent a localized region of the NE containing NPCs with reduced Nup intensity, perhaps representing partial NPC disassembly or assembly intermediates.

Multiple lines of evidence suggest that the reduced Nup intensities near the SPBs is the result of physical exclusion of NPCs from this region. First, we observed similar exclusion patterns for multiple Nups including members of each NPC subcomplex (Fig. 5F). The size of the exclusion zone was relatively stable for all Nups, although was slightly larger for components of the nuclear basket (Fig. 5F). The majority of Nups were reduced by $\approx 50 \%$ over the SPB-proximal region, although the structural components Nup97 and Nup37 were excluded to a lesser extent (Fig. 5F, inset). If the observed Nup exclusion was due to changes in NPC composition near the SPB, we expected to see a relationship between NPC/Nup intensity and proximity to the SPBs. However, the intensities for individual NPCs that were proximal (within $100 \mathrm{~nm}$ ra- dius of SPBs) and those that were distal were equivalent for multiple Nups, and only marginally $(\approx 20 \%)$ reduced for a subset of Nups (Fig. 5G, Fig. S3). Collectively, these findings support a model in which the reduced Nup intensity surrounding the SPBs is the result of reduced presence of NPCs in this region, rather than localized alterations of NPC composition.

\section{Exclusion of NPCs near the SPBs requires Lem2-mediated centromere tethering. We recently} showed that the INM protein Lem2 localizes to the SPB during interphase and forms a ring with similar dimensions to that of the NPC exclusion zone $(86,92)$ (Fig. 6A). We hypothesized that the Lem 2 ring may be a component of the physical barrier that prevents NPCs from localizing to this region. Indeed, deletion of lem $2+$ resulted in a significant decrease in NPC exclusion from the SPB region (Fig. 6B) without affecting NPC composition, as SPB proximal and distal Nup intensities were similar in lem $2 \Delta$ mutants. A decrease in NPC exclusion was not seen in cells lacking the INM protein Ima1 or the second $S$. pombe LEM domain-containing protein, Man1, that does not localize to the SPB (92) (Fig. 6B).

Lem2 contains two nucleoplasmic regions: an N-terminal $\mathrm{HEH} / \mathrm{LEM}$ domain that is required for DNA binding and centromere tethering at the SPB $(93,94)$, and a C-terminal 
bioRxiv preprint doi: https://doi.org/10.1101/2021.08.11.455278; this version posted August 11, 2021. The copyright holder for this preprint (which was not certified by peer review) is the author/funder, who has granted bioRxiv a license to display the preprint in perpetuity. It is made available under aCC-BY 4.0 International license.

A
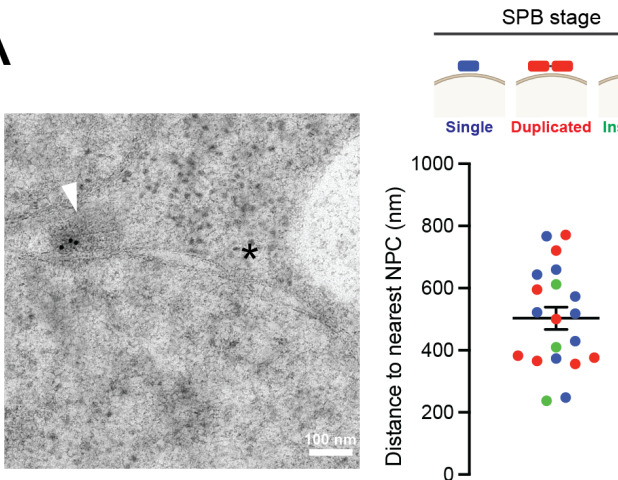

C
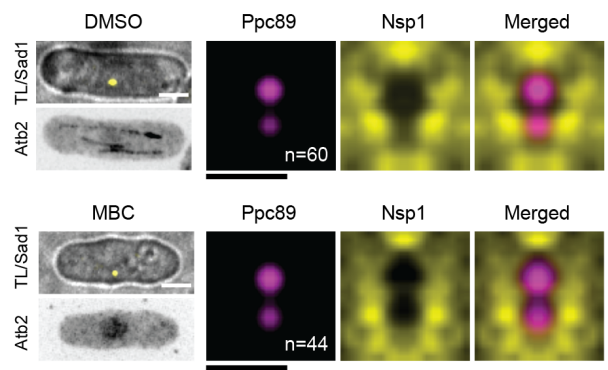

Ppc89

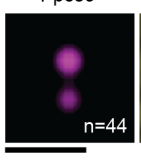

Nsp1

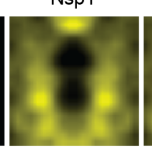

Merged

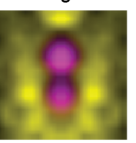

$\mathrm{F}$

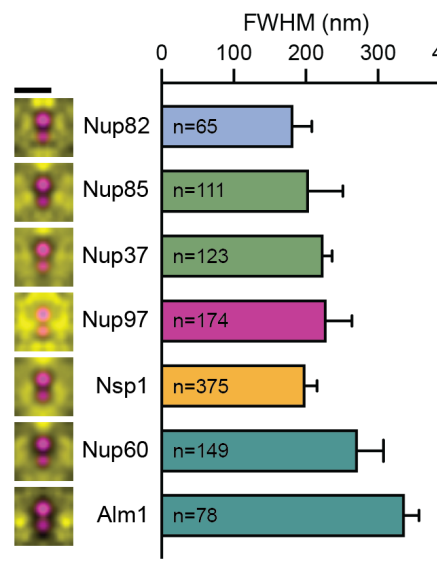

400

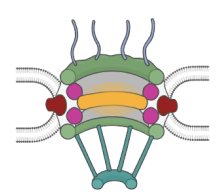

Reduction in intensity inside exclusion zone $\begin{array}{lll}0.0 & 0.5 & 1.0\end{array}$

Nup82-
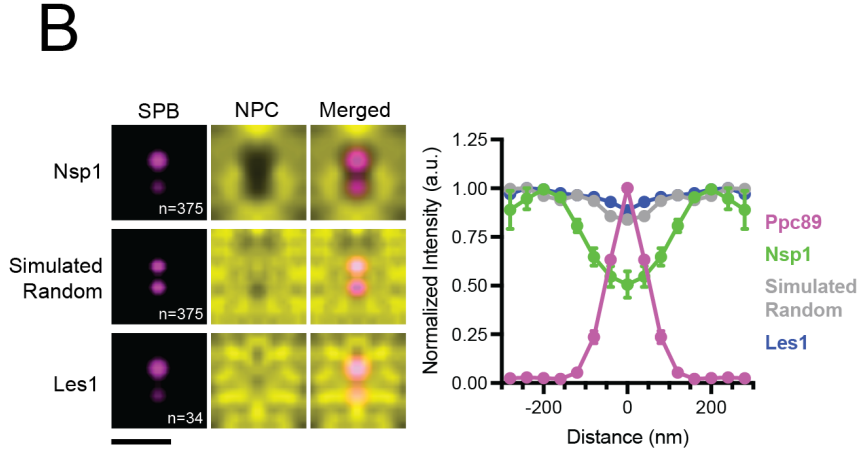

$\mathrm{D}$

$E$

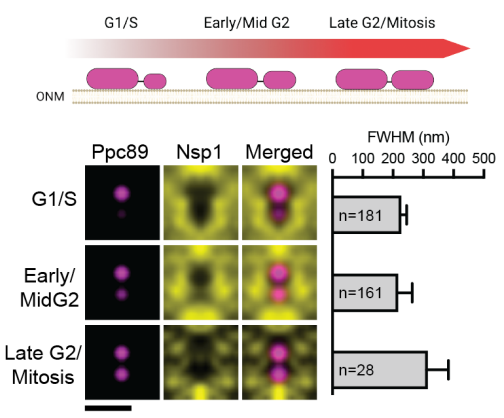

Anaphase B

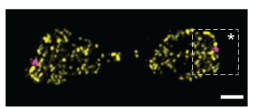

Max. Proj. Middle Slice

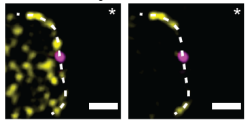

Averaged, $n=35$

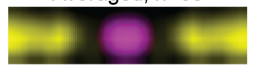

G

Nsp1-GFP, 3650 NPCs

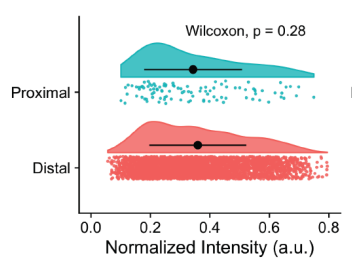

Nup97-GFP, 1527 NPCs

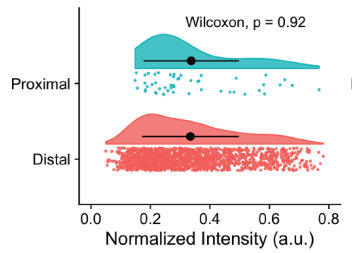

Nup85-GFP, 1157 NPCs

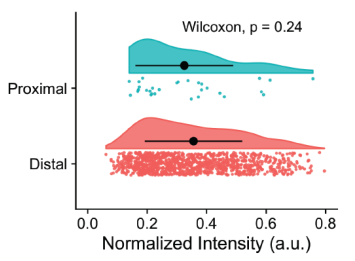

Alm1-GFP, 485 NPCs

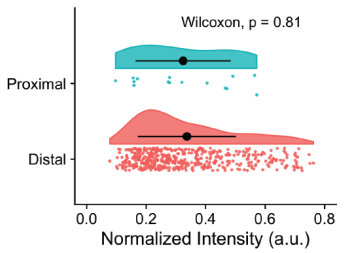

Fig. 5. NPC exclusion from the SPB proximal region of the NE A) ImmunoEM image of SPB component Ppc1-GFP (arrowhead). The nearest NPC is highlighted with asterisk. Plot of distance from SPB to the nearest NPC, based on SPB stage: blue = single SPB; red = duplicated SPB; green = inserted SPB. B) SPA-SIM images of Ppc89mCh (magenta) and Nsp1-GFP (NPCs), Les1-GFP and simulated random distributions (yellow). Normalized intensity profiles across the mother and daughter SPBs. Error bars, SD. Bar, $0.5 \mu \mathrm{m}$. C) Confocal image for microtubules (mCh-Atb2) and SPBs (Sad1-GFP) in cells treated with DMSO (control) or $25 \mu \mathrm{g} / \mathrm{mL}$ MBC for $1 \mathrm{~h}$ to depolymerize microtubules. Bar, $3 \mu \mathrm{m}$. SPA-SIM images of Ppc89-mCh (magenta) and Nsp1-GFP (yellow) in these cells. Bar, $0.5 \mu \mathrm{m}$. D) Schematic of SPB duplication through the cell cycle. SPA-SIM images of Ppc89-mCh (magenta) and Nsp1-GFP (yellow) based on daughter/mother Ppc89-mCh intensity ratios (G1/S, 0.5; Early/Mid G2, 0.5 - 0.8; Late G2/Mitosis, 0.8). Plot of FWHM of Nsp1 exclusion zone for each stage. Error bars, $95 \%$ Cl. Bar, $0.5 \mu \mathrm{m}$. E) 3D-SIM projection of Nsp1-GFP (yellow) and Ppc89-mCh (magenta) in anaphase. Bar, $1 \mu \mathrm{m}$. Enlarged images of SPB region, showing maximum projection and single middle z-slice. Bar, $0.5 \mu \mathrm{m}$. Averaged image of Nsp1-GFP NPCs relative to SPB in mitotically dividing nuclei (see Methods). Bar, $0.5 \mu \mathrm{m}$. F) SPA-SIM images of GFP-tagged Nups (yellow) and Ppc89-mCh SPBs (magenta), along with FWHM plot as in D. The majority of Nups have $\approx 50 \%$ reduction in intensity near the SPB relative to the surrounding NE. Bar, $0.5 \mu$ m. G) Individual data points and kernel-smoothed density distributions of Nup-GFP intensities for NPC foci that were proximal $(<100 \mathrm{~nm})$ or distal $(>100 \mathrm{~nm})$ to the SPB. Nup intensities for proximal and distal NPCs were compared using a non-parametric, unpaired Wilcoxon rank sum test. Black dots represent the mean normalized intensity value, and error bars show SD. 
bioRxiv preprint doi: https://doi.org/10.1101/2021.08.11.455278; this version posted August 11, 2021. The copyright holder for this preprint (which was not certified by peer review) is the author/funder, who has granted bioRxiv a license to display the preprint in perpetuity. It is made available under aCC-BY 4.0 International license.

A

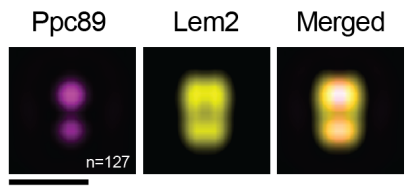

Overlay of Lem2 and Nsp1 SPA-SIM data sets

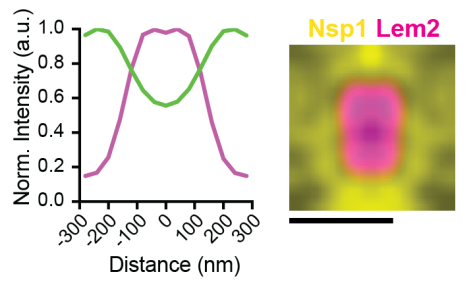

C
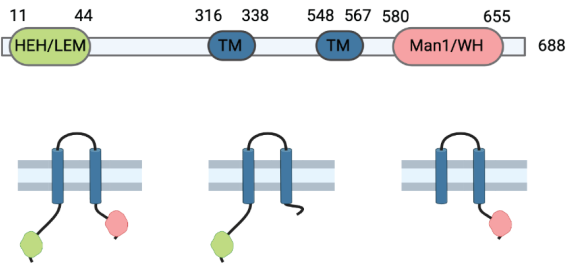

lem $2^{\Delta c}$

(1-567)

lem2 $2^{\Delta N}$

$(316-688)$
B

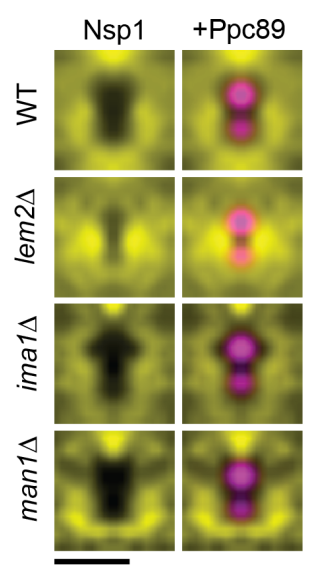

D
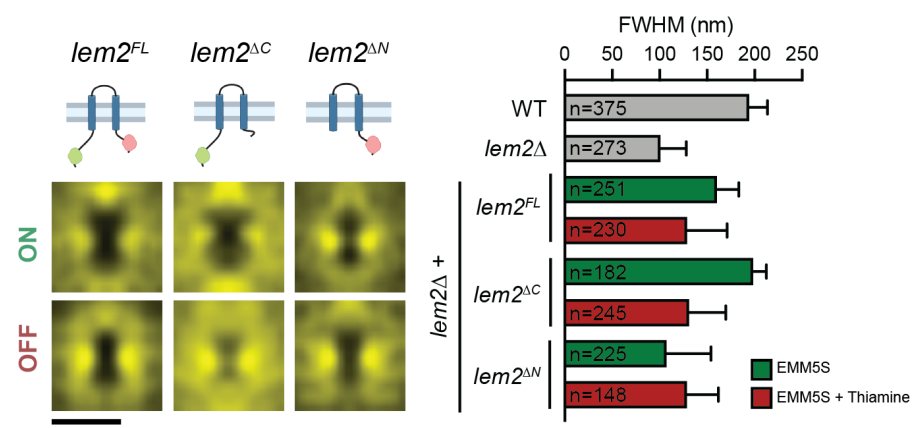

$\mathrm{E}$
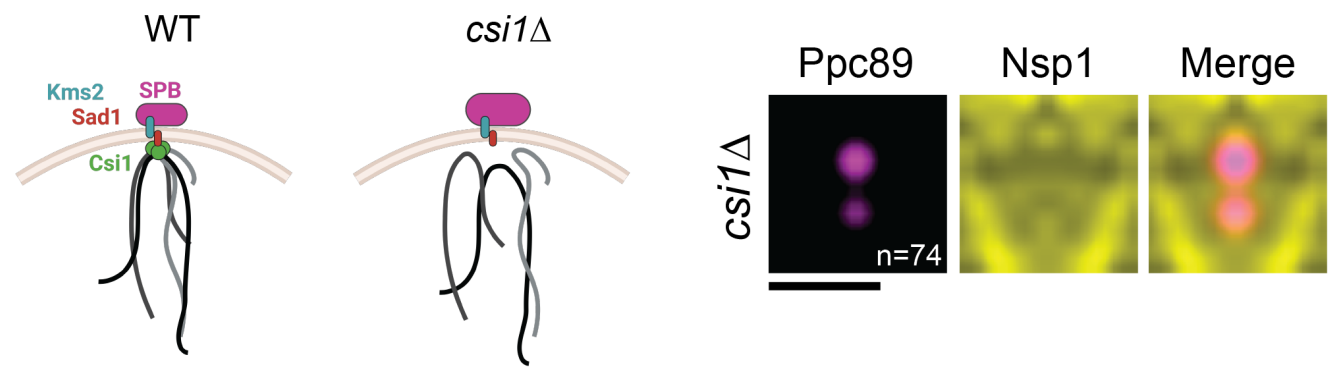

Fig. 6. NPC exclusion requires Lem2 and centromere tethering A) SPA-SIM of Lem2-GFP (yellow) and Ppc89-mCh (magenta). N, number of averaged images. Lem2GFP and Ppc89-mCh intensity profiles are shown below. Overlay of SPA-SIM datasets for Lem2-GFP (magenta) and Nsp1-GFP (yellow). Bars, 0.5 $\mu$ m. B) SPA-SIM

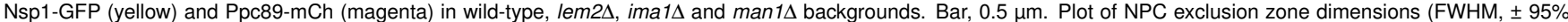
$\mathrm{Cl}$ ). Nsp1-GFP intensity in SPB proximal and distal regions was plotted and compared using a Wilcoxon rank sum test. Black dots represent the mean normalized intensity value, and error bars show SD. C) Schematic of Lem2, including rescue constructs. D) SPA-SIM images of Nsp1-GFP (yellow) in lem2 $2 \Delta$ cells with lem2 $2^{F L}$, lem2 ${ }^{\Delta C}$ or lem2 $2^{\Delta N}$ constructs turned on or off. Bar, $0.5 \mu \mathrm{m}$. Plot of NPC exclusion zone dimensions (FWHM, $\pm 95 \% \mathrm{Cl}$ ). For comparison, wild-type and lem2 $\Delta$ dimensions from (B) are also shown. F) Schematic of centromere tethering at the SPB in wild-type and csi1 $\Delta$ cells. SPA-SIM of Nsp1-GFP (yellow) and Ppc89-mCh (magenta) in csi1 cells. N, number of averaged images. Bar, $0.5 \mu \mathrm{m}$.

Man1/Winged-Helix domain that tethers telomeres to the nuclear periphery (95) (Fig. 6C). Lem2 truncation mutants lacking the N- and C-termini localize to the SPB (94), allowing us to test which regions of Lem2 are needed for NPC exclusion. Full-length or mutant versions of Lem2 were stably integrated at the ura4+ locus and expressed as Cterminal $3 \times \mathrm{xH}$ fusion proteins in a lem $2 \Delta$ background using the thiamine-regulatable $n m t 41$ promoter system $(96,97)$ (Fig. 6D, Fig. S4). Exclusion of NPCs from the SPB region was similar to wild-type cells when either full-length $\left(\right.$ lem $\left.2^{F L}\right)$ or $l e m 2^{\Delta C}$ constructs were expressed (EMM5S). However, lem $2^{\Delta N}$ expression resulted in an exclusion zone FWHM similar to lem $2 \Delta$ mutants (Fig. 6D), suggesting that NPC exclusion depends on the function of Lem2's DNAbinding N-terminal HEH/LEM domain.

The size of the NPC exclusion zone was reduced in lem $2 \Delta$ and $l e m 2^{\Delta N}$ strains, however, NPCs were still strongly excluded from a smaller region directly underneath the SPBs. During interphase, fission yeast centromeres tether under the SPBs (98) through interactions with multiple proteins includ- 
ing Lem2, Sad1, and Csi1 (reviewed in (91). We hypothesized that the smaller exclusion zone observed in the absence of Lem2 could reflect a physical barrier formed by the remaining NE-centromere interactions. To test whether tethering of centromeres to the SPBs drives exclusion of NPCs from this smaller region, we examined NPC exclusion in csil $\Delta$ mutants, in which $\approx 70 \%$ of cells exhibit defects in centromere tethering (99). Interestingly, in csil $\Delta$ cells, NPCs were no longer excluded from the SPB-proximal region (Fig. 6E). This supports a model whereby the exclusion of NPCs from the SPB region is the result of physical interactions between centromeres and INM proteins, including Lem2, that tether the centromeres under the SPBs during interphase.

\section{Forcing NPCs into the SPB proximal region delays mi-} totic progression. A key question is why NPCs are excluded from the region near the SPB in $S$. pombe: is this important for SPB insertion into the NE during mitosis, for SPB separation or for some unknown aspect of SPB biology? Although lem $2 \Delta$ and csi $1 \Delta$ cells lose NPC exclusion at the SPB, these mutants likely have pleiotropic effects due to their multiple functions in nuclear size regulation, SPB function and chromosome organization $(95,99-104)$. Therefore, we developed an ectopic system to forcibly tether NPCs at the SPB (Fig. 7A). When expressed in mitotically growing cells, the INM protein Bqt1 localizes to the SPB through interactions with the INM protein Sad1 (93). Expression of Bqt1-mCh fused to GFP-binding protein (Bqt1-mCh-GBP) using a thiamine-repressible promoter $(n m t 3 X)$ forced the recruitment of NPCs containing Nsp1-GFP (a component of both the central channel and the nuclear basket (105), into the SPB proximal zone, reducing the width of the exclusion zone by $\approx 50 \%$ (Fig. 7B). Forced localization of NPCs into the SPB proximal region caused a moderate impairment in growth (Fig. 7C). Using live cell imaging to visualize SPBs and microtubules in the presence and absence of Bqt1mediated NPC tethering, we showed that this growth delay is likely the result of two defects. First, the time between the depolymerization of interphase microtubules and the formation of $3 \mu \mathrm{m}$ metaphase spindle (106) was increased by $\approx 20 \%$ when NPCs were tethered (Fig. 7D). Second, NPC tethering caused spindle orientation defects, with prophase and metaphase spindles having an average deviation of $\approx 30$ degrees from the longitudinal axis of the cell compared to $\approx 18$ degrees when the Bqt1-GBP tether was repressed (Fig. 7E). Tethering did not affect microtubule nucleation at the SPB, including the formation of cytoplasmic microtubules. Together, these data suggest an unexpected role for a zone of NPC exclusion at the SPB that is important for mitotic progression.

\section{Discussion}

Multiple imaging approaches, including EM and fluorescence microscopy, have been used to determine the number and distribution of NPCs in various systems. The higher resolution afforded by EM and super-resolution light microscopy methods often comes at a price of significant increases in the time required for sample preparation, image acquisition and analysis. In contrast, 3D-SIM generates highresolution datasets using standard fluorescence microscopy approaches, allowing for quantitative analysis of NPC organization through whole nuclei. We apply 3D-SIM to fission yeast nuclei to provide the first map of NPCs in this system. We find that NPC density in S. pombe nuclei is similar to that described for many metazoan nuclei and is constant over a range of nuclear sizes. This finding has important implications regarding the mechanisms used for NPC assembly in fission yeast. For example, the total number of NPCs present in the two daughter nuclei in late mitosis is $\approx 26 \%$ greater than the number present in the mother nucleus prior to division. In agreement with previous findings (67), we observed that although the combined nuclear volume of the daughter nuclei is similar to the total volume of the mother nucleus, the combined surface area is $\approx 34 \%$ greater than that of the mother nucleus (Table S1). Together, this suggests that NPC assembly continues to occur during the rapid expansion of the NE during cell division (107). However, NE expansion during mitosis takes place over a time frame of roughly 20$25 \mathrm{~min}$, significantly shorter than the 45-60 min required for completion of NPC assembly in budding yeast and during interphase in metazoans $(29,108)$. During the short cell cycles of the syncytial nuclear divisions in Drosophila embyros, rapid NPC assembly occurs via incorporation of assembly intermediates from annulate lamellae $(109,110)$. However, by EM and by fluorescence microscopy, we and others do not observe pools of NPCs/Nups outside the NE so it is unclear how $S$. pombe maintains NPC density during mitosis. Continued NPC assembly in $c d c 25.22$ arrested cells that have low Cdk1 activity suggests that unlike in metazoans, Cdk1 is not required for NPC assembly in $S$. pombe. The fact that the nuclear size increase and NPC assembly in $c d c 25.22$ were blocked by inhibiting fatty acid synthesis supports a model for negative regulation of NPC assembly similar to that proposed in vertebrates mediated by Tpr/ERK. In this model, signals emanating from existing NPCs inhibit assembly of NPCs in the surrounding region and inhibition of NPC assembly can be overcome by reducing NPC density through NE expansion. Spk1, the fission yeast ERK ortholog, is not essential for cell viability (111), suggesting that mechanistic details of this pathway may differ between species.

A major benefit of the 3D-SIM approach is that the improved resolution allowed for identification of distinct patterns of organization for clustered NPCs in $S$. pombe. NPC clusters were often observed to be organized in ring-like patterns $\approx 250-300 \mathrm{~nm}$ in diameter that were more prevalent in the clustering mutants nupl $32 \Delta$ and nem $1 \Delta$. These rings are smaller than typical yeast autophagosomes (300-900 nm diameter) $(112,113)$. although they are similar in size to nuclear-derived vesicles containing NPCs seen in EM images of NPCs being removed by autophagy in budding yeast (114). Our observation of increased ring number by 3D-SIM when nup $132 \Delta$ cells were grown on solid instead of liquid 
bioRxiv preprint doi: https://doi.org/10.1101/2021.08.11.455278; this version posted August 11, 2021. The copyright holder for this preprint (which was not certified by peer review) is the author/funder, who has granted bioRxiv a license to display the preprint in perpetuity. It is made available under aCC-BY 4.0 International license.

A

\section{EMM5S + Thiamine}

Tethering OFF

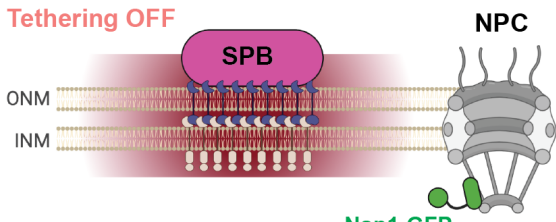

EMM5S

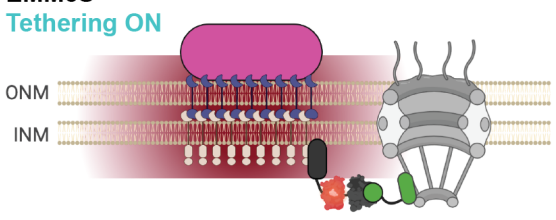

Bqt1-mCh-GBP
B

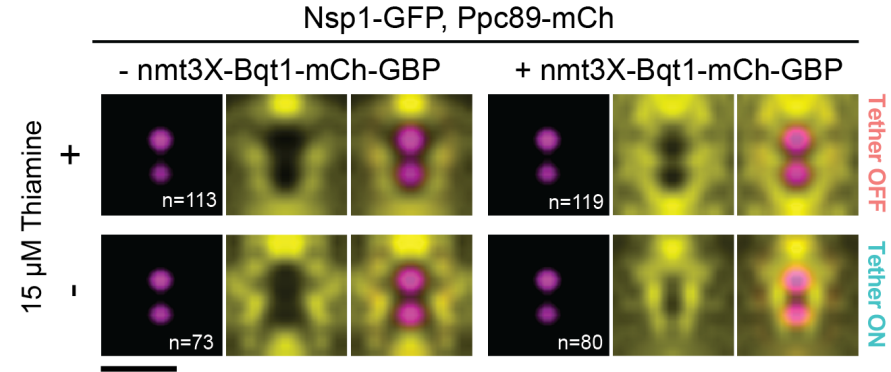

FWHM (nm)

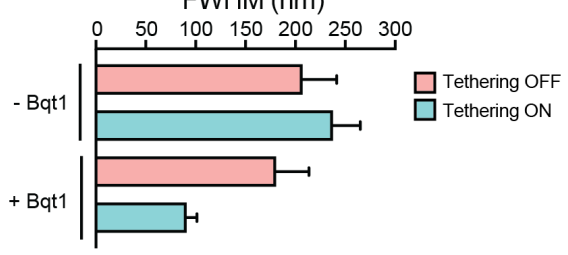

C

EMM5S + $15 \mu M$ Thiamine Tethering OFF

Nsp1-GFP/Ppc89-mCh

nmt3X-Bqt1-mCh-GBP

Nsp1-GFP/Ppc89-mCh + nmt3X-Bqt1-mCh-GBP
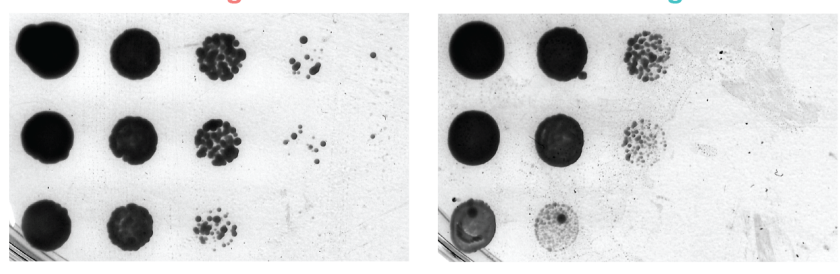

D
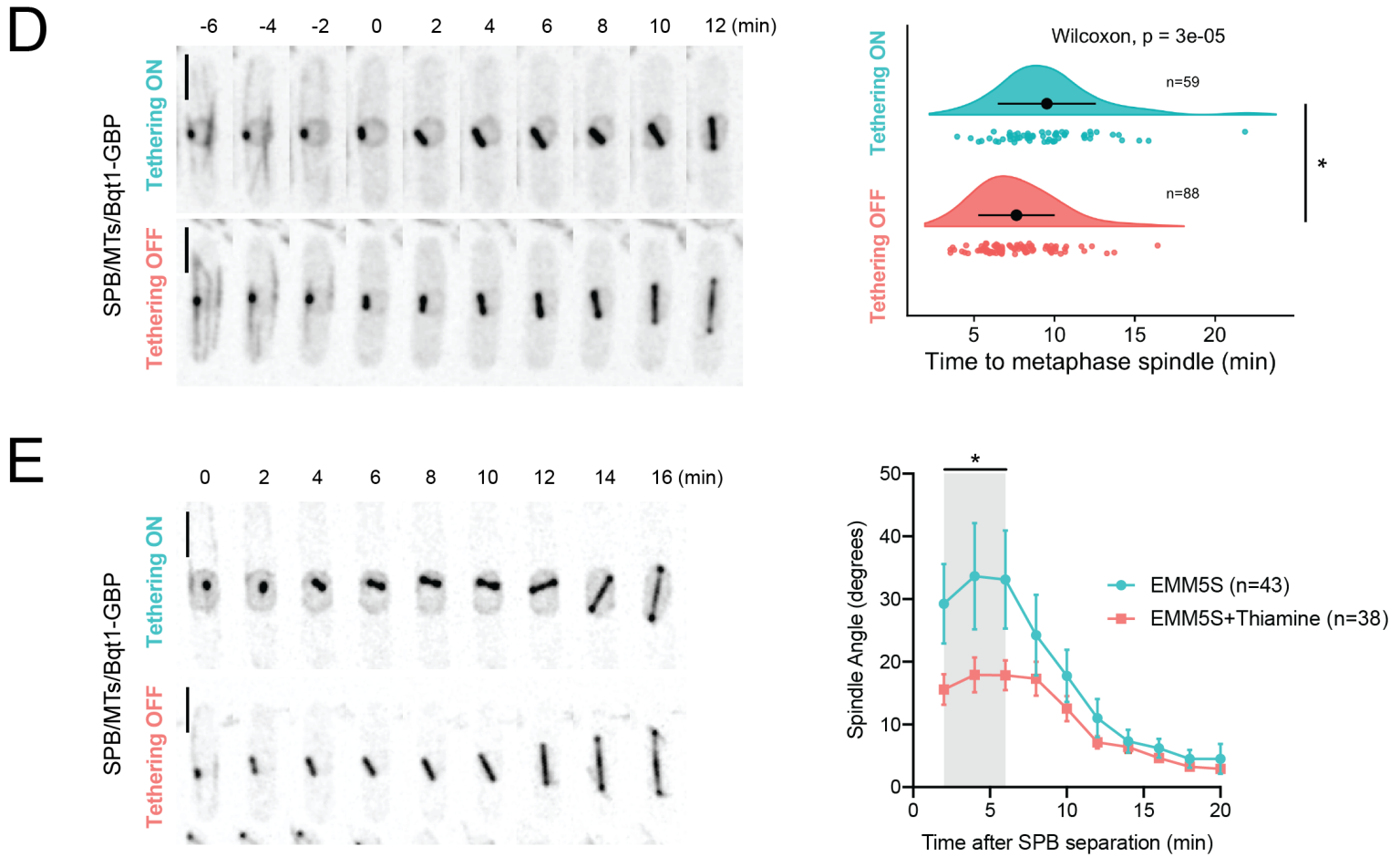

Fig. 7. Loss of NPC exclusion at the SPB delays mitotic progression A) Schematic of NPC tethering to the SPB. Bqt1-mCh-GBP localizes to the SPB and recruits NPCs via Nsp1-GFP into the SPB proximal region (red gradient). Bqt1-mCh-GBP expression is repressed by addition of thiamine to the media. B) Cells with or without Bqt1-mCh-GBP were grown in the presence (off) or absence (on) of thiamine. SPA-SIM images of Ppc89-mCh (magenta) and Nsp1-GFP (yellow). Bar, 0.5 $\mu$ m. n, number of images. Dimensions of the NPC exclusion zone in each condition are shown below. Error bars represent $95 \% \mathrm{Cl}$ of SD of Gaussian fit to the Nsp1-GFP intensity profile. C) Growth of the indicated cells was tested at $30^{\circ} \mathrm{C}$. D-E) Time-lapse imaging of Nsp1-GFP/Bqt1-mCh-GBP cells containing Ppc89-mCh (SPB) and mCh-Atb2 (microtubules). Time points are indicated above, with $t=0$ based on cytoplasmic microtubule disassembly. Bar, $5 \mu \mathrm{m}$. D) The time from cytoplasmic microtubule disassembly to formation of a metaphase spindle ( $3 \mu \mathrm{m}$ ) was analyzed and is shown on the right. $\mathrm{N}$, number of cells analyzed. Statistical significance $\left.{ }^{*}\right)$ was determined using a Wilcoxon rank sum test. Black dots represent the mean and error bars show SD. E) Plot of average spindle angle relative to the cell length axis versus time after SPB separation for the indicated number of cells $(\mathrm{n})$. Error bars, SD. Timepoints highlighted in gray with asterisk determined to be significantly different between conditions using unpaired t-test with Welch correction. 
media suggests that changes in nutrient availability triggers NPC reorganization into ring clusters of a consistent size. Whether the formation of these rings promotes their subsequent removal via autophagy or other pathways remains to be tested. However, the increased frequency of ring clusters in nup $132 \Delta$ cells may provide insights into the mechanism driving their formation. Nup132 is a structural Nup that facilitates interactions between the structural scaffold of the NPC with lipids through its N-terminal ALPS motif $(115,116)$. Deletion of Nup132 may alter the interactions between NPCs and specific lipid species present in the NE, making nup $132 \Delta$ cells especially sensitive to changes in lipid composition that may occur due to nutrient availability during growth on plates.

Time-lapse imaging of NPC clusters revealed two strikingly different approaches to how clustered NPCs are handled during mitosis. In neml $\Delta$ mutants, both excess nuclear membrane material and NPCs are segregated into the anaphase bridge region during nuclear division (Fig. 3F), a distinct nuclear compartment that is a unique site of NPC disassembly $(70,71)$. Clusters of NPCs formed in $t t s 1 \Delta$ cells specifically during mitotic NE expansion also localized to the anaphase bridge (117). This suggests that the anaphase bridge region of the NE may serve as a site where NPCs and NE material are sent to be removed during division, analogous to the NE-derived compartment that forms in budding yeast meiosis II to sequester and degrade NPCs $(57,118)$. The fact that nup $132 \Delta$ clusters do not similarly localize to the anaphase bridge suggests that the fate of NPC clusters likely depends on the mechanisms driving the clustering. If nup $132 \Delta$ clusters interact with specific lipids, this may also explain their portioning with the SPB, which has been proposed to contain a unique NE (119) (below).

Our results clearly demonstrate that the region of the NE over the nucleolus and near the SPB are distinct from other NE regions. It is not surprising that the nucleolar region has reduced NPC density and pores lacking the basket Nups Alm1 and Nup211 given similar observations of NPC number and composition in plants, mammals and fungi $(12,35,36)$. In contrast, we were somewhat surprised to see reduced NPC density at the SPB in fission yeast given that NPC density is increased near SPBs in budding yeast $(27,45)$. Perhaps this is reflective of differences in the roles NPCs play in SPB insertion into the NE in the two fungi - NPCs are thought to facilitate SPB incorporation into the NE in S. cerevisiae but do not appear to be required for SPB assembly into the NE in S. pombe. Moreover, our data indicate that tethering of NPCs into the SPB proximal region in fission yeast delays the formation of a properly oriented metaphase spindle. This phenotype may be due in part to the artificial tether, although it is important to note that csil $\Delta$ mutants, which lose NPC exclusion, also have mitotic defects $(100,120)$.

A key question is how populations of distinct NPC composition are established and maintained in specific regions of the NE, since fungal NPCs laterally diffuse through the NE. At least three potential models exist: intact NPCs diffuse into a subregion and are partially disassembled; a unique NPC subpopulation is assembled in that region of the NE; or subregions of the NE have unique properties and preferentially allow for NPCs of specific composition to diffuse in and/or be retained. Partial disassembly of fungal NPCs has been reported in multiple cell types and conditions (121-123). However, the fact that Nup exclusion is decreased at the SPB when NPCs are artificially tethered suggests that an SPB-derived signal such as phosphorylation by a SPB localized kinase likely does not induce NPC remodeling in this region of the NE. Instead, we favor a model for physical NPC exclusion from the SPB involving both centromeres and Lem2. At the $\mathrm{SPB}$, tethering of centromeres to INM-localized SPB components forms a physical barrier that prevents the diffusion of NPCs through the NE into the SPB proximal region. NPCs that contain a basket may be particularly affected as they are likely to associate with chromatin and other complexes through this structure. Consistent with the steric model, if we reduced or eliminated centromere tethering, either by removing Lem2's N-terminal HEH/LEM domain or by deletion of csil+, the NPC exclusion zone was diminished. Interestingly, NPCs remain excluded from the SPB region throughout mitosis, including during periods where Lem 2 no longer localizes to the SPB (92). During these stages, NPC exclusion is likely maintained by multiple proteins that form SPBring structures during mitosis, including Imal and Sad1 (86).

It is likely that the reduced NPC density and altered basket composition over the nucleolus is produced through a different mechanism. In budding yeast, the NE over the nucleolus is more amenable to membrane expansion than regions outside of the nucleolus (124). Similar differences in NE membrane properties over the nucleolus may exist in S. pombe and could drive the observed NPC heterogeneity. For example, differences in membrane composition or fluidity could alter the ability for NPCs to diffuse laterally through this portion of the NE, leading to reduced density over the nucleolus. Alternatively, the region over the nucleolus could have higher rates of NE membrane incorporation and NPC assembly. In this scenario, the reduced presence of Alm1 and Nup211 could be due to these Nups being the last components added during NPC assembly (108). In either case, the reduced presence of Alm1/Nup211 over the nucleolus could be the result of interactions between chromatin and NPCs containing Alm1/Nup211 (either directly or indirectly via basketassociated complexes involved in mRNA processing and export) that may prevent their diffusion back into the nucleolarfacing NE compartment. Our results establish $S$. pombe as a model for further studies determining the mechanisms that establish and maintain distinct populations of heterogeneous NPC composition within single nuclei. Importantly, our results demonstrate that the reduced NPC density and specific loss of Tpr-ortholog basket components over the nucleolus is not unique to budding yeast, but is a conserved feature of nuclear organization across highly divergent species. The ability for 3D-SIM to resolve and quantify individual NPCs labelled with multiple fluorescent proteins at endogenous levels provides tools to begin to interrogate how altered NPC compositions may allow for functional specialization of NPC 
function at distinct regions of the NE.

\section{Methods}

A. Yeast strains and plasmids. All S. pombe strains used in this manuscript are listed in Table S2. Deletion strains were obtained from the $S$. pombe haploid deletion library (Bioneer). Genes of interest were endogenously tagged using standard PCR-based methods (125), with lithium acetate transformation and colony selection as previously described (126). Cells were cultured in yeast extract with supplements (YES) media $(5 \mathrm{~g}$ yeast extract, $30 \mathrm{~g}$ dextrose, 0.2 $\mathrm{g}$ each adenine, uracil, histidine, leucine and lysine, in $1 \mathrm{~L}$ of water) at $25^{\circ} \mathrm{C}$, unless otherwise noted. For experiments using the $n m t 41+$ or $n m t 3 x$ promoters, cells were cultured in Edinburgh minimal media with amino acid supplements (EMM5S) (127) at $30^{\circ} \mathrm{C}$. Thiamine was added to EMM5S to a final concentration of $15 \mu \mathrm{M}$ for $18-24 \mathrm{~h}$ at $30^{\circ} \mathrm{C}$ to repress expression. All strains were maintained in liquid culture for at least $48 \mathrm{~h}$ with back diluting to maintain cultures in logarithmic growth prior to imaging, unless otherwise noted. For growth assays, $4 \mathrm{OD}_{600}$ of logarithmically growing cells were serially diluted ten-fold and spotted onto EMM5S agar plates $\left(+/-15 \mu \mathrm{M}\right.$ thiamine) at $30^{\circ} \mathrm{C}$. Where noted, cultures were treated with methyl benzimidazol-2-yl carbamate (MBC, 25 $\mu \mathrm{g} / \mathrm{mL}$ ), Cerulenin $(10 \mu \mathrm{M})$ or dimethylsulfoxide (DMSO, vehicle control).

The coding sequence, or subdomains, for lem $2+$ was amplified from genomic DNA using HiFi PCR master mix (Clontech) and cloned into NdeI/Xhol-digested pREP41-MCS+. The resulting plasmid was used as a template to amplify nmt41-lem2-3xHA, which was transformed into the ura4+ locus of lem $2 \Delta$ cells as described (128). Subdomains were similarly cloned and integrated. Integration was verified by PCR, and thiamine-dependent repression was validated by immuno blotting of whole cell extracts using anti-HA antibodies (Roche, 3F10).

B. NPC quantitation and analysis by 3D-SIM. Exponentially growing cells were collected by centrifugation for $3 \mathrm{~min}$ at $3000 \mathrm{rcf}$ and fixed in a solution of $4 \%$ formaldehyde supplemented with $200 \mathrm{mM}$ glucose. Fixed cells were imaged in phosphate buffered saline, $\mathrm{pH} 7.4$ with an Applied Precision OMX Blaze V4 (GE Healthcare) equipped with a 60x 1.42 NA Olympus Plan Apo oil objective and two PCO Edge sCMOS cameras. Two-color (GFP/mCherry) imaging was performed using 488-nm (GFP) or 561-nm (mCherry) lasers with alternating excitation, and a 405/488/561/640 dichroic with 504-552-nm and 590-628-nm emission filters. Images were acquired over a volume covering the entire nucleus with z-spacing of 125-nm (typically $4 \mu \mathrm{m}$ ). Widefield images of NPC clusters were acquired using the same microscope and settings as above but operating in widefield mode. SIM images were reconstructed with Softworx (Applied Precision Ltd), with a Wiener filter of 0.001. Except where noted, SIM images shown throughout are maximum intensity projections of all z-slices, scaled using bilinear interpolation with linear brightness and contrast adjustments in ImageJ (129).

Image analysis was performed using a number of custom plugins and macros for ImageJ/FIJI, all of which are freely available at http://research.stowers. org/imagejplugins/. Additional documentation and source code used for NPC density analysis can be found at http://www. stowers.org/research/ publications/libpb-1640. Statistical analysis was performed using R or Graph Pad Prism v 9.0. Average values along with standard deviation (SD) from the mean are shown based on the indicated number of samples (n), unless otherwise noted.

To quantitate the number of NPCs, individual nuclei were detected and segmented in an automated fashion using custom ImageJ plugins. Briefly, maximum intensity projections were used to perform automatic local thresholding for nuclear segmentation using a semi-automated protocol allowing the user to add and remove missed or poorly segmented ROIs. Each nucleus was cropped, and NPCs were detected using a "track max not mask" approach, in which the brightest voxel in the image is found and a spheroid with a diameter of 8 pixels $(320 \mathrm{~nm})$ in $\mathrm{x}$ and $\mathrm{y}$ and 5 slices in $\mathrm{z}(625 \mathrm{~nm})$ is masked around that voxel. This process repeats until no voxels remain above a minimum threshold of $25 \%$ of the maximum intensity in the image. After NPC detection, the threedimensional coordinates were used to model the NE surface using the "convhulln" function from the geometry package in R. Occasionally we observed the presence of points detected away from the NE (representing noise or foci of cytoplasmic signal). To remove these points prior to computation of the convex hull, we included an optimization step in which up to ten percent of the initial points could be removed if doing so increased the fraction of points present on the convex hull surface. Surface area and volume metrics were extracted for the 3D convex hull and used to derive NPC density values.

For a secondary method to validate density measurements, the nuclei image stacks were sum projected and background subtracted by selecting an ROI adjacent to the nucleus using the plugin "roi average subtract jru v1". The resulting images were used to measure the nucleus area either by automated thresholding or occasionally by manual tracing of the nucleus in cases where thresholding failed to reliably segment the nucleus. The nucleus ROI areas and integrated densities were then extracted using "Analyze Particles" in ImageJ.

Cells were sorted into cell cycle stages using the following criteria: early $\mathrm{G} 2$, cell length $<9.5 \mu \mathrm{m}$, mononucleate; mid-G2, length between 9.5 and $11 \mu \mathrm{m}$, mononucleate; late G2/early mitosis, length $>=11.0 \mu \mathrm{m}$, mononucleate; late mitosis, length $>=11 \mu \mathrm{m}$, binucleate; G1/S, septated.

Single particle analysis was performed as previously described (85). Briefly, mother and daughter SPB spots were manually selected, and each spot was fitted to two 3D Gaussian functions and realigned along the axis between these two functions. To allow for visualization of NPC distributions in the $\mathrm{x}-\mathrm{y}$ plane relative to the SPBs, a Euclidean distance filter was used in ImageJ to select for images in which both SPB 
points were at least $400 \mathrm{~nm}$ away from the edge of the nucleus based on maximum intensity projections. Realigned images were averaged as described previously $(84,85)$. To account for biologically irrelevant directional bias in the $\mathrm{x}-\mathrm{y}$ plane, the averaged images were further averaged with a mirrored (x-y) image. All averaged images presented were thresholded to display pixels above a threshold of $25 \%$ of the maximum intensity value. Quantitation of Nup intensity relative to the mother and daughter SPB was performed in an automated manner, using line profiles with a width (12 pixels) covering both mother and daughter SPBs to generate profiles of the Nup/GFP intensities. Proteins of interest were considered to be excluded if the normalized peak intensity fell below 0.8 , based on the reduction in signal observed in simulated random datasets. For excluded proteins, the plot profiles were fit to a Gaussian and the width of the exclusion zone was measured by computing the full-width half-maximal (FWHM) value for the fit curve using the equation $\mathrm{FWHM}=2.355$ $\mathrm{x}$ standard deviation (SD). The size of exclusion zones for proteins of interest were compared using the FWHM value from plot profiles of the averaged SPA-SIM images, with error bars representing the $95 \%$ confidence interval of the SD of the Gaussian fit. Curve fitting and statistical analyses were performed using GraphPad Prism v. 9.0.

An analogous approach was used to generate an averaged image for Nups relative to the SPB in anaphase and telophase nuclei. First, nuclei in these stages were identified in which the SPB was clearly observed within the NE from single zslices. These slices were then used to manually trace the NE (based on Nup signal), followed by straightening of the polyline using the ImageJ macro "polyline profile jru v1" with a line width of 8 pixels and the option for "Output Straightened" selected. The resulting straightened images were used to identify the location of the SPB, and a $1 \mu \mathrm{m}$ region of the image centered on the SPB was cropped. The cropped images were then combined, and an averaged image was generated. The averaged image was further averaged with a mirrored image (in both horizontal and vertical directions) to generate a final averaged image with no directional biases.

C. Simulations and modeling. For comparison of SPASIM data to the distribution expected to be observed by random chance, we simulated spherical nuclei with a of radius $1.25 \mu \mathrm{m}$ (based on average dimensions of mid-G2 stage nuclei) with 125 randomly positioned NPCs to model an NPC density $\left(6.4 \mathrm{NPCs} / \mu \mathrm{m}^{2}\right)$ similar to the average NPC density observed by 3D-SIM. NPCs were simulated as 3D Gaussians with a FWHM in the x-y plane of $100 \mathrm{~nm}$ and $300 \mathrm{~nm}$ in z, a minimum center-to-center distance of $100 \mathrm{~nm}$ and a maximum intensity of 100 photons. The simulated pixel size was $40 \mathrm{~nm}$ with a z-slice spacing of $125 \mathrm{~nm}$. The resulting intensities were multiplied by 20 (artificial "Gain") and a Gaussian read noise with a standard deviation of 40 intensity units was added to each voxel. For simulation of SPA-SIM data, two SPB points were simulated with a center-to-center distance of $180 \mathrm{~nm}$ in a second channel. Simulated images were processed using the same approaches outlined above for NPC quantitation and SPA-SIM analyses.

D. Confocal imaging. Confocal imaging to determine NPC density over the nucleolus was performed in log-phase cells expressing the nucleolar protein, Nuc1-mCherry, and utilized a PerkinElmer UltraVIEW VoX with a Yokogawa CSU-X1 spinning disk head, a 100x 1.46 NA Olympus Plan Apo oil objective and CCD (ORCA-R2) and EMCCD (C9100-13) cameras. GFP/mCherry images were taken using a 488-

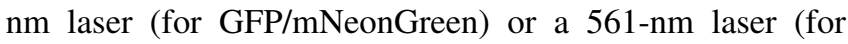
mCherry), with alternating excitation. Images were collected using the Volocity imaging software with a $\mathrm{z}$ spacing of 0.3 $\mu \mathrm{m}$ over a volume of $8 \mu \mathrm{m}$. To assess nucleoporin intensity levels at the nuclear envelope, the middle four slices of the image stack were sum projected using ImageJ, and the nuclear envelope was manually traced to generate line profiles for both GFP (Nup) and mCherry (nucleolus) channels in nuclei where the nucleolus was oriented to one side of the nucleus in the $x-y$ direction. The line profiles for Nuc1-mCherry were boxcar smoothened, thresholded at their half-maximal values, and all profiles were aligned at the center of the Nuc1mCherry peak. The nucleoporin line profiles were then resampled and averaged to generate average intensity profiles. Mean normalized intensity values for the Nup signal at the center of the nucleolar peak were calculated from three independent biological replicates and plotted in GraphPad Prism v. 9.0.

To analyze spindle morphology and NPC cluster dynamics in live cells, $\approx 200-300 \mu \mathrm{L}$ of $\log$ phase cells were applied to $35-\mathrm{mm}$ glass bottom dishes (MaTek, no. 1.5 coverslip) that had been pre-coated with $1 \mathrm{mg} / \mathrm{mL}$ soybean lectin (in water) for $15 \mathrm{~min}$ and rinsed with YES media. After cells were allowed to settle for $30 \mathrm{~min}$ at $30^{\circ} \mathrm{C}, 2 \mathrm{~mL}$ of pre-warmed YES media was carefully added. Cells were imaged on a Nikon Ti-E microscope equipped with a CSI W1 spinning disk (Yokogawa) using a 60x 1.4 NA Olympus Plan Apo oil objective and an iXon DU897 Ultra EMCCD (Andor) camera. GFP and mCherry were excited at $488 \mathrm{~nm}$ and 561 $\mathrm{nm}$, respectively, and collected through ET525/36m (GFP) or ET605/70m (mCherry) bandpass filters. Samples were maintained at $30^{\circ} \mathrm{C}$ using an Oko Lab stage top incubator. Images were acquired over a $6 \mu \mathrm{m}$ volume with $0.3 \mu \mathrm{m} \mathrm{z}$-spacing for $45 \mathrm{~min}$ at 2 min intervals.

SPB distances were measured using the Euclidean distance between manually annotated SPBs from maximum intensity projections of the full image stacks. Spindle orientations were determined manually using the Angle tool in ImageJ to measure the difference of the spindle relative to the cell axis from maximum intensity projected image stacks. To generate image montages presented in figures, maximum intensity projections of the full image stacks were background subtracted, bleach corrected (using the Simple Ratio method) and scaled 3-fold ( $\mathrm{x}$ and $\mathrm{y}$ ) using bilinear interpolation.

E. Electron microscopy. The distance between SPBs and the nearest NPC was measured in images from samples prepared as previously described (85). Sections in which both 
bioRxiv preprint doi: https://doi.org/10.1101/2021.08.11.455278; this version posted August 11, 2021. The copyright holder for this preprint (which was not certified by peer review) is the author/funder, who has granted bioRxiv a license to display the preprint in perpetuity. It is made available under aCC-BY 4.0 International license.

E Electron microscopy

the outer and inner nuclear membranes were clearly resolved were used for analysis. The distance from the center of the SPB to the nearest NPC (determined based on visible fusion of the INM and ONM) was measured by manually tracing the nuclear envelope using the polyline tool in ImageJ, and the data was plotted in GraphPad Prism v. 9.0.

\section{ACKNOWLEDGEMENTS}

We thank Julie Cooper for strains, Zulin Yu and members of the Stowers Microscopy Core Facility for imaging assistance, and Brian Slaughter and members of the Jaspersen lab for discussion and feedback on this manuscript. Schematics throughout were created using BioRender.com. Research reported in this publication was supported by the Stowers Institute for Medical Research and NIH-NIGMS under award number R01GM121443 (to SLJ). J.M.V is a recipient of a Ruth L. Kirschstein NRSA Postdoctoral Fellowship (F32GM133096). Original data underlying this manuscript can be downloaded from the Stowers Original Data Repository at http://www.stowers.org/research/publications/libpb-1640. The authors declare no competing financial interests.

\section{DATA AVAILABILITY STATEMENT}

Upon peer-reviewed publication, all strains generated for and used in this study will be made available upon reasonable request. Original data underlying this manuscript will be accessible at the Stowers Original Data Repository at $h t t p$ : //www.stowers.org/research/publications/libpb-1640.

\section{Bibliography}

1. Daniel H. Lin and André Hoelz. The Structure of the Nuclear Pore Complex (An Update). Annual Review of Biochemistry, 88:725-783, June 2019. ISSN 1545-4509. doi: 10.1146/ annurev-biochem-062917-011901.

2. Pau Pascual-Garcia and Maya Capelson. The nuclear pore complex and the genome: organizing and regulatory principles. Current Opinion in Genetics \& Development, 67 142-150, April 2021. ISSN 1879-0380. doi: 10.1016/j.gde.2021.01.005.

3. Marcela Raices and Maximiliano A D'Angelo. Nuclear pore complexes and regulation of gene expression. Current Opinion in Cell Biology, 46:26-32, June 2017. ISSN 09550674. doi: $10.1016 /$ j.ceb.2016.12.006.

4. Frank Alber, Svetlana Dokudovskaya, Liesbeth M. Veenhoff, Wenzhu Zhang, Julia Kipper, Damien Devos, Adisetyantari Suprapto, Orit Karni-Schmidt, Rosemary Williams, Brian T. Chait, Andrej Sali, and Michael P. Rout. The molecular architecture of the nuclear pore complex. Nature, 450(7170):695-701, November 2007. ISSN 1476-4687. doi: 10.1038/ nature06405.

5. Martin Beck and Ed Hurt. The nuclear pore complex: understanding its function through structural insight. Nature Reviews Molecular Cell Biology, 18(2):73-89, February 2017. ISSN 1471-0072. doi: 10.1038/nrm.2016.147.

6. Daniel Stoffler, Bernhard Feja, Birthe Fahrenkrog, Jochen Walz, Dieter Typke, and Ueli Aebi. Cryo-electron Tomography Provides Novel Insights into Nuclear Pore Architecture: Implications for Nucleocytoplasmic Transport. Journal of Molecular Biology, 328(1):119130, April 2003. ISSN 00222836. doi: 10.1016/S0022-2836(03)00266-3.

7. M. Beck. Nuclear Pore Complex Structure and Dynamics Revealed by Cryoelectron Tomography. Science, 306(5700):1387-1390, November 2004. ISSN 0036-8075, 10959203. doi: 10.1126/science.1104808.

8. Seung Joong Kim, Javier Fernandez-Martinez, llona Nudelman, Yi Shi, Wenzhu Zhang, Barak Raveh, Thurston Herricks, Brian D. Slaughter, Joanna A. Hogan, Paula Upla, Ilan E. Chemmama, Riccardo Pellarin, Ignacia Echeverria, Manjunatha Shivaraju, Azraa S Chaudhury, Junjie Wang, Rosemary Williams, Jay R. Unruh, Charles H. Greenberg, Erica Y. Jacobs, Zhiheng Yu, M. Jason de la Cruz, Roxana Mironska, David L. Stokes, John D. Aitchison, Martin F. Jarrold, Jennifer L. Gerton, Steven J. Ludtke, Christopher W. Akey, Brian T. Chait, Andrej Sali, and Michael P. Rout. Integrative structure and functional anatomy of a nuclear pore complex. Nature, March 2018. ISSN 1476-4687. doi: 10.1038/nature26003.

9. Alexander von Appen, Jan Kosinski, Lenore Sparks, Alessandro Ori, Amanda L. DiGuilio, Benjamin Vollmer, Marie-Therese Mackmull, Niccolo Banterle, Luca Parca, Panagiotis Kastritis, Katarzyna Buczak, Shyamal Mosalaganti, Wim Hagen, Amparo Andres-Pons, Edward A. Lemke, Peer Bork, Wolfram Antonin, Joseph S. Glavy, Khanh Huy Bui, and Martin Beck. In situ structural analysis of the human nuclear pore complex. Nature, 526 (7571):140-143, October 2015. ISSN 1476-4687. doi: 10.1038/nature15381.

10. Shyamal Mosalaganti, Jan Kosinski, Sahradha Albert, Miroslava Schaffer, Daniela Strenkert, Patrice A. Salomé, Sabeeha S. Merchant, Jürgen M. Plitzko, Wolfgang Baumeister, Benjamin D. Engel, and Martin Beck. In situ architecture of the algal nuclear pore complex. Nature Communications, 9(1):2361, December 2018. ISSN 2041-1723. doi: 10.1038/s41467-018-04739-y.

11. Christian E Zimmerli, Matteo Allegretti, Vasileios Rantos, Sara K Goetz, Agnieszka Obarska-Kosinska, levgeniia Zagoriy, Aliaksandr Halavatyi, Julia Mahamid, Jan Kosinski, and Martin Beck. Nuclear pores constrict upon energy depletion. July 2020. doi: 10.1101/2020.07.30.228585.

12. G. G. Maul. The nuclear and the cytoplasmic pore complex: structure, dynamics, distribution, and evolution. International Review of Cytology. Supplement, (6):75-186, 1977. ISSN 0074-770X.

13. G. G. Maul and L. Deaven. Quantitative determination of nuclear pore complexes in cycling cells with differing DNA content. The Journal of Cell Biology, 73(3):748-760, June 1977. ISSN 0021-9525, 1540-8140. doi: 10.1083/jcb.73.3.748.

14. L. M. Garcia-Segura, M. Lafarga, M. T. Berciano, P. Hernandez, and M. A. Andres. Distribution of nuclear pores and chromatin organization in neurons and glial cells of the rat cerebellar cortex. The Journal of Comparative Neurology, 290(3):440-450, December 1989. ISSN 0021-9967, 1096-9861. doi: 10.1002/cne.902900311.

15. G. G. Maul, L. L. Deaven, J. J. Freed, G. L. Campbell, and W. Beçak. Investigation of the determinants of nuclear pore number. Cytogenetics and Cell Genetics, 26(2-4):175-190, 1980. ISSN 0301-0171.

16. B. Czerniak, L. G. Koss, and A. Sherman. Nuclear pores and DNA ploidy in human bladder carcinomas. Cancer Research, 44(9):3752-3756, September 1984. ISSN 0008-5472.

17. Stephen Sakuma, Marcela Raices, Joana Borlido, Valeria Guglielmi, Ethan Y. S. Zhu, and Maximiliano A. D'Angelo. Inhibition of Nuclear Pore Complex Formation Selectively Induces Cancer Cell Death. Cancer Discovery, 11(1):176-193, January 2021. ISSN 21598290. doi: 10.1158/2159-8290.CD-20-0581.

18. L. M. García-Segura, G. Olmos, P. Tranque, P. Aguilera, and F. Naftolin. Nuclear pores in rat hypothalamic arcuate neurons: sex differences and changes during the oestrous cycle. Journal of Neurocytology, 16(4):469-475, August 1987. ISSN 0300-4864.

19. M. Carmo-Fonseca. Testosterone-induced changes in nuclear pore complex number of prostatic nuclei from castrated rats. Journal of Ultrastructure Research, 80(2):243-251, August 1982. ISSN 0022-5320.

20. H. E. Ortiz and J. C. Cavicchia. Androgen-induced changes in nuclear pore number and in tight junctions in rat seminal vesicle epithelium. The Anatomical Record, 226(2):129-134, February 1990. ISSN 0003-276X. doi: 10.1002/ar.1092260202.

21. G. G. Maul, H. M. Maul, J. E. Scogna, M. W. Lieberman, G. S. Stein, B. Y. Hsu, and T. W. Borun. Time sequence of nuclear pore formation in phytohemagglutinin-stimulated lymphocytes and in HeLa cells during the cell cycle. The Journal of Cell Biology, 55(2): 433-447, November 1972. ISSN 0021-9525.

22. M. C. Many, J. F. Denef, A. C. Cordier, and S. Haumont. Morphometry of nuclear pore complexes in thyroid cells during hyperplasia and involution. Molecular and Cellular Endocrinology, 23(2):137-149, August 1981. ISSN 0303-7207.

23. Jeffrey N. Savas, Brandon H. Toyama, Tao Xu, John R. Yates, and Martin W. Hetzer. Extremely long-lived nuclear pore proteins in the rat brain. Science (New York, N.Y.), 335 (6071):942, February 2012. ISSN 1095-9203. doi: 10.1126/science. 1217421.

24. Maximiliano A. D'Angelo, Marcela Raices, Siler H. Panowski, and Martin W. Hetzer. AgeDependent Deterioration of Nuclear Pore Complexes Causes a Loss of Nuclear Integrity in Postmitotic Cells. Cell, 136(2):284-295, January 2009. ISSN 0092-8674. doi: 10.1016/ j.cell.2008.11.037.

25. Brandon H. Toyama, Jeffrey N. Savas, Sung Kyu Park, Michael S. Harris, Nicholas T. Ingolia, John R. Yates, and Martin W. Hetzer. Identification of Long-Lived Proteins Reveals Exceptional Stability of Essential Cellular Structures. Cell, 154(5):971-982, August 2013. ISSN 0092-8674. doi: 10.1016/j.cell.2013.07.037.

26. Shotaro Otsuka and Jan Ellenberg. Mechanisms of nuclear pore complex assembly two different ways of building one molecular machine. FEBS Letters, 592(4):475-488, February 2018. ISSN 0014-5793, 1873-3468. doi: 10.1002/1873-3468.12905.

27. Mark Winey, Defne Yarar, Thomas H. Giddings, and David N. Mastronarde. Nuclear Pore Complex Number and Distribution throughout the Saccharomyces cerevisiae Cell Cycle by Three-Dimensional Reconstruction from Electron Micrographs of Nuclear Envelopes. Molecular Biology of the Cell, 8(11):2119-2132, November 1997. ISSN 1059-1524.

28. Kazuhiro Maeshima, Haruki lino, Saera Hihara, and Naoko Imamoto. Nuclear size, nuclear pore number and cell cycle. Nucleus (Austin, Tex.), 2(2):113-118, April 2011. ISSN 19491042. doi: 10.4161/nucl.2.2.15446.

29. Shotaro Otsuka, Khanh Huy Bui, Martin Schorb, M. Julius Hossain, Antonio Z. Politi, Birgit Koch, Mikhail Eltsov, Martin Beck, and Jan Ellenberg. Nuclear pore assembly proceeds by an inside-out extrusion of the nuclear envelope. eLife, 5:e19071, September 2016. ISSN 2050-084X. doi: 10.7554/eLife.19071.

30. Elisa Dultz and Jan Ellenberg. Live imaging of single nuclear pores reveals unique assembly kinetics and mechanism in interphase. The Journal of Cell Biology, 191(1):15-22, October 2010. ISSN 0021-9525, 1540-8140. doi: 10.1083/jcb.201007076.

31. Kazuhiro Maeshima, Haruki lino, Saera Hihara, Tomoko Funakoshi, Ai Watanabe, Masaomi Nishimura, Reiko Nakatomi, Kazuhide Yahata, Fumio Imamoto, Tsutomu Hashikawa, Hideo Yokota, and Naoko Imamoto. Nuclear pore formation but not nuclear growth is governed by cyclin-dependent kinases (Cdks) during interphase. Nature Structural \& Molecular Biology, 17(9):1065-1071, September 2010. ISSN 1545-9985. doi: 10.1038/nsmb.1878.

32. Asako McCloskey, Arkaitz Ibarra, and Martin W. Hetzer. Tpr regulates the total number of nuclear pore complexes per cell nucleus. Genes \& Development, 32(19-20):1321-1331, October 2018. ISSN 0890-9369, 1549-5477. doi: 10.1101/gad.315523.118.

33. Mark Kittisopikul, Takeshi Shimi, Meltem Tatli, Joseph Riley Tran, Yixian Zheng, Ohad Medalia, Khuloud Jaqaman, Stephen A. Adam, and Robert D. Goldman. Computational analyses reveal spatial relationships between nuclear pore complexes and specific lamins. Journal of Cell Biology, 220(4):e202007082, April 2021. ISSN 0021-9525, 1540-8140. doi: 10.1083/jcb.202007082.

34. Charlene Boumendil, Priya Hari, Karl C. F. Olsen, Juan Carlos Acosta, and Wendy A Bickmore. Nuclear pore density controls heterochromatin reorganization during senescence. Genes \& Development, 33(3-4):144-149, February 2019. ISSN 1549-5477. doi: 10.1101/gad.321117.118.

35. N.J. Severs, E.G. Jordan, and D.H. Williamson. Nuclear pore absence from areas of close association between nucleus and vacuole in synchronous yeast cultures. Journal of Ultrastructure Research, 54(3):374-387, March 1976. ISSN 00225320. doi: 10.1016/ S0022-5320(76)80023-8.

36. L. F. La Cour and B. Wells. Nuclear pores at prophase of meiosis in plants. Philosophical Transactions of the Royal Society of London. Series B, Biological Sciences, 268(891):95100, July 1974. ISSN 0962-8436. doi: 10.1098/rstb.1974.0017.

37. N. Harris. Nuclear pore distribution and relation to adjacent cytoplasmic organelles in cotyledon cells of developing Vicia faba. Planta, 141(2):121-128, January 1978. ISSN 
bioRxiv preprint doi: https://doi.org/10.1101/2021.08.11.455278; this version posted August 11, 2021. The copyright holder for this preprint (which was not certified by peer review) is the author/funder, who has granted bioRxiv a license to display the preprint in perpetuity. It is made available under aCC-BY 4.0 International license.

0032-0935. doi: 10.1007/BF00387877.

38. M.L. Miller, A. Andringa, and L. Hastings. Relationships between the nuclear membrane, nuclear pore complexes, and organelles in the type II pneumocyte. Tissue and Cell, 27(6): 613-619, December 1995. ISSN 00408166. doi: 10.1016/S0040-8166(05)80017-5.

39. Renjie Wang, Alain Kamgoue, Christophe Normand, Isabelle Léger-Silvestre, Thomas Mangeat, and Olivier Gadal. High resolution microscopy reveals the nuclear shape of budding yeast during cell cycle and in various biological states. J Cell Sci, page jcs.188250, January 2016. ISSN 0021-9533, 1477-9137. doi: 10.1242/jcs.188250.

40. Xiaozhou Pan, Paul Roberts, Yan Chen, Erik Kvam, Natalyia Shulga, Kristen Huang, Sandra Lemmon, and David S. Goldfarb. Nucleus-Vacuole Junctions in Saccharomyces cerevisiae Are Formed Through the Direct Interaction of Vac8p with Nvj1p. Molecular Biology of the Cell, 11(7):2445-2457, July 2000. ISSN 1059-1524, 1939-4586. doi: 10.1091/mbc.11.7.2445.

41. Robert Peter Aaronson and Günter Blobel. ON THE ATTACHMENT OF THE NUCLEAR PORE COMPLEX. Journal of Cell Biology, 62(3):746-754, September 1974. ISSN 15408140, 0021-9525. doi: 10.1083/jcb.62.3.746.

42. R. P. Aaronson and G. Blobel. Isolation of nuclear pore complexes in association with a lamina. Proceedings of the National Academy of Sciences, 72(3):1007-1011, March 1975. ISSN 0027-8424, 1091-6490. doi: 10.1073/pnas.72.3.1007.

43. Nathalie Daigle, Joël Beaudouin, Lisa Hartnell, Gabriela Imreh, Einar Hallberg, Jennifer Lippincott-Schwartz, and Jan Ellenberg. Nuclear pore complexes form immobile networks and have a very low turnover in live mammalian cells. Journal of Cell Biology, 154(1): 71-84, July 2001. ISSN 1540-8140, 0021-9525. doi: 10.1083/jcb.200101089.

44. Kazuhiro Maeshima, Kazuhide Yahata, Yoko Sasaki, Reiko Nakatomi, Taro Tachibana, Tsutomu Hashikawa, Fumio Imamoto, and Naoko Imamoto. Cell-cycle-dependent dynamics of nuclear pores: pore-free islands and lamins. Journal of Cell Science, 119(21): 4442-4451, November 2006. ISSN 0021-9533, 1477-9137. doi: 10.1242/jcs.03207.

45. Diana Rüthnick, Annett Neuner, Franziska Dietrich, Daniel Kirrmaier, Ulrike Engel, Michael Knop, and Elmar Schiebel. Characterization of spindle pole body duplication reveals a regulatory role for nuclear pore complexes. Journal of Cell Biology, 216(8):2425-2442, August 2017. ISSN 0021-9525, 1540-8140. doi: 10.1083/jcb.201612129.

46. Caterina Strambio-de Castillia, Günter Blobel, and Michael P. Rout. Proteins Connecting the Nuclear Pore Complex with the Nuclear Interior. The Journal of Cell Biology, 144(5): 839-855, March 1999. ISSN 0021-9525.

47. Vincent Galy, Olivier Gadal, Micheline Fromont-Racine, Alper Romano, Alain Jacquier, and Ulf Nehrbass. Nuclear Retention of Unspliced mRNAs in Yeast Is Mediated by Perinuclear Mlp1. Cell, 116(1):63-73, January 2004. ISSN 0092-8674. doi: 10.1016/S0092-8674(03) 01026-2

48. Jin-Ah Bae, Donggerami Moon, and Jin Ho Yoon. Nup211, the fission yeast homolog of Mlp1/Tpr, is involved in mRNA export. Journal of Microbiology (Seoul, Korea), 47(3): 337-343, June 2009. ISSN 1976-3794. doi: 10.1007/s12275-009-0125-7.

49. P. Bangs, B. Burke, C. Powers, R. Craig, A. Purohit, and S. Doxsey. Functional analysis of Tpr: identification of nuclear pore complex association and nuclear localization domains and a role in mRNA export. The Journal of Cell Biology, 143(7):1801-1812, December 1998. ISSN 0021-9525. doi: 10.1083/jcb.143.7.1801.

50. Mario Niepel, Kelly R. Molloy, Rosemary Williams, Julia C. Farr, Anne C. Meinema, Nicholas Vecchietti, lleana M. Cristea, Brian T. Chait, Michael P. Rout, and Caterina Strambio-De-Castillia. The nuclear basket proteins Mlp1p and Mlp2p are part of a dynamic interactome including Esc1p and the proteasome. Molecular Biology of the Cell, 24 (24):3920-3938, December 2013. ISSN 1059-1524. doi: 10.1091/mbc.E13-07-0412.

51. Silvia Salas-Pino, Paola Gallardo, Ramón R. Barrales, Sigurd Braun, and Rafael R. Daga. The fission yeast nucleoporin Alm1 is required for proteasomal degradation of kinetochore components. J Cell Biol, page jcb.201612194, October 2017. ISSN 0021-9525, 15408140. doi: $10.1083 /$ jcb. 201612194

52. Paolo Colombi, Brant M. Webster, Florian Fröhlich, and C. Patrick Lusk. The transmission of nuclear pore complexes to daughter cells requires a cytoplasmic pool of Nsp1. The Journal of Cell Biology, 203(2):215-232, October 2013. ISSN 1540-8140. doi: 10.1083/ jcb.201305115.

53. Tadashi Makio, Diego L. Lapetina, and Richard W. Wozniak. Inheritance of yeast nuclear pore complexes requires the Nsp1p subcomplex. The Journal of Cell Biology, 203(2): 187-196, October 2013. ISSN 1540-8140. doi: 10.1083/jcb.201304047.

54. Zhanna Shcheprova, Sandro Baldi, Stephanie Buvelot Frei, Gaston Gonnet, and Yves Barral. A mechanism for asymmetric segregation of age during yeast budding. Nature, 454(7205):728-734, August 2008. ISSN 1476-4687. doi: 10.1038/nature07212.

55. Barbara Boettcher, Tatiana T. Marquez-Lago, Mathias Bayer, Eric L. Weiss, and Yves Barral. Nuclear envelope morphology constrains diffusion and promotes asymmetric protein segregation in closed mitosis. The Journal of Cell Biology, 197(7):921-937, June 2012. ISSN 0021-9525. doi: 10.1083/jcb.201112117.

56. Arun Kumar, Priyanka Sharma, Mercè Gomar-Alba, Zhanna Shcheprova, Anne Daulny, Trinidad Sanmartín, Irene Matucci, Charlotta Funaya, Miguel Beato, and Manuel Mendoza. Daughter-cell-specific modulation of nuclear pore complexes controls cell cycle entry during asymmetric division. Nature Cell Biology, page 1, March 2018. ISSN 1476-4679. doi: 10.1038/s41556-018-0056-9.

57. Grant A King, Jay S Goodman, Jennifer G Schick, Keerthana Chetlapalli, Danielle M Jorgens, Kent L McDonald, and Elçin Ünal. Meiotic cellular rejuvenation is coupled to nuclear remodeling in budding yeast. eLife, 8:e47156, August 2019. ISSN 2050-084X. doi: 10.7554/eLife.47156.

58. Haruhiko Asakawa, Tomoko Kojidani, Chie Mori, Hiroko Osakada, Mamiko Sato, Da-Qiao Ding, Yasushi Hiraoka, and Tokuko Haraguchi. Virtual Breakdown of the Nuclear Envelope in Fission Yeast Meiosis. Current Biology, 20(21):1919-1925, November 2010. ISSN 09609822. doi: 10.1016/j.cub.2010.09.070.

59. Charlotte Capitanchik, Charles R. Dixon, Selene K. Swanson, Laurence Florens, Alastair R. W. Kerr, and Eric C. Schirmer. Analysis of RNA-Seq datasets reveals enrichment of tissue-specific splice variants for nuclear envelope proteins. Nucleus (Austin, Tex.), 9(1): 410-430, 2018. ISSN 1949-1042. doi: 10.1080/19491034.2018.1469351.

60. Alessandro Ori, Niccolò Banterle, Murat Iskar, Amparo Andrés-Pons, Claudia Escher, Huy
Khanh Bui, Lenore Sparks, Victor Solis-Mezarino, Oliver Rinner, Peer Bork, Edward A. emke, and Martin Beck. Cell type-specific nuclear pores: a case in point for contextdependent stoichiometry of molecular machines. Molecular Systems Biology, 9:648, 2013. ISSN 1744-4292. doi: 10.1038/msb.2013.4.

61. Maximiliano A. D'Angelo, J. Sebastian Gomez-Cavazos, Arianna Mei, Daniel H. Lackner, and Martin W. Hetzer. A change in nuclear pore complex composition regulates cell differentiation. Developmental Cell, 22(2):446-458, February 2012. ISSN 1878-1551. doi: 10.1016/j.devcel.2011.11.021.

62. Melissa Kane, Stephanie V Rebensburg, Matthew A Takata, Trinity M Zang, Masahiro Yamashita, Mamuka Kvaratskhelia, and Paul D Bieniasz. Nuclear pore heterogeneity influences HIV-1 infection and the antiviral activity of MX2. eLife, 7:e35738, August 2018. ISSN 2050-084X. doi: 10.7554/eLife.35738.

63. J. Sebastian Gomez-Cavazos and Martin W. Hetzer. The nucleoporin gp210/Nup210 controls muscle differentiation by regulating nuclear envelope/ER homeostasis. Journal of Cell Biology, 208(6):671-681, March 2015. ISSN 1540-8140, 0021-9525. doi: 10.1083/jcb.201410047.

64. Valeria Guglielmi, Stephen Sakuma, and Maximiliano A. D'Angelo. Nuclear pore complexes in development and tissue homeostasis. Development (Cambridge, England), 147 (23):dev183442, December 2020. ISSN 1477-9129. doi: 10.1242/dev.183442.

65. Javier Fernandez-Martinez and Michael P. Rout. One Ring to Rule them All? Structura and Functional Diversity in the Nuclear Pore Complex. Trends in Biochemical Sciences, 46(7):595-607, July 2021. ISSN 0968-0004. doi: 10.1016/j.tibs.2021.01.003.

66. Qing Yang, Michael P Rout, and Christopher W Akey. Three-Dimensional Architecture of the Isolated Yeast Nuclear Pore Complex: Functional and Evolutionary Implications. Molecular Cell, 1(2):223-234, January 1998. ISSN 10972765. doi: 10.1016/ S1097-2765(00)80023-4.

67. Frank R. Neumann and Paul Nurse. Nuclear size control in fission yeast. The Journal of Cell Biology, 179(4):593-600, November 2007. ISSN 0021-9525. doi: 10.1083/jcb. 200708054

68. Agnes Grallert, Andrea Krapp, Steve Bagley, Viesturs Simanis, and lain M. Hagan. Recruitment of NIMA kinase shows that maturation of the S. pombe spindle-pole body occurs over consecutive cell cycles and reveals a role for NIMA in modulating SIN activity. Genes \& Development, 18(9):1007-1021, May 2004. ISSN 0890-9369, 1549-5477. doi: 10.1101/gad.296204.

69. Rafael Lucena, Noah Dephoure, Steve P. Gygi, Douglas R. Kellogg, Victor A. Tallada, Rafael R. Daga, and Juan Jimenez. Nucleocytoplasmic transport in the midzone membrane domain controls yeast mitotic spindle disassembly. The Journal of Cell Biology, 209 (3):387-402, May 2015. ISSN 0021-9525, 1540-8140. doi: 10.1083/jcb.201412144.

70. Gautam Dey, Siân Culley, Scott Curran, Uwe Schmidt, Ricardo Henriques, Wanda Kukulski, and Buzz Baum. Closed mitosis requires local disassembly of the nuclear envelope. Nature, 585(7823):119-123, September 2020. ISSN 0028-0836, 1476-4687. doi: 10.1038/s41586-020-2648-3.

71. María Expósito-Serrano, Ana Sánchez-Molina, Paola Gallardo, Silvia Salas-Pino, and Rafael R. Daga. Selective Nuclear Pore Complex Removal Drives Nuclear Envelope Division in Fission Yeast. Current Biology, 30(16):3212-3222.e2, August 2020. ISSN 09609822. doi: 10.1016/j.cub.2020.05.066.

72. H. Moor and K. Mühlethaler. FINE STRUCTURE IN FROZEN-ETCHED YEAST CELLS. The Journal of Cell Biology, 17(3):609-628, June 1963. ISSN 0021-9525.

73. P. Russell and P. Nurse. Negative regulation of mitosis by wee1+, a gene encoding a protein kinase homolog. Cell, 49(4):559-567, May 1987. ISSN 0092-8674. doi: 10.1016/ 0092-8674(87)90458-2.

74. Paul Nurse, Pierre Thuriaux, and Kim Nasmyth. Genetic control of the cell division cycle in the fission yeast Schizosaccharomyces pombe. Molecular and General Genetics MGG, 146(2):167-178, January 1976. ISSN 0026-8925, 1432-1874. doi: 10.1007/BF00268085.

75. T. Yorimitsu and D. J. Klionsky. Autophagy: molecular machinery for self-eating. Cell Death \& Differentiation, 12(S2):1542-1552, 2005. ISSN 1350-9047 doi: 10.1038/sj.cdd.4401765.

76. Siau Wei Baï, Jacques Rouquette, Makoto Umeda, Wolfgang Faigle, Damarys Loew, Shelley Sazer, and Valérie Doye. The Fission Yeast Nup107-120 Complex Functionally Interacts with the Small GTPase Ran/Spi1 and Is Required for mRNA Export, Nuclear Pore Distribution, and Proper Cell Division. Molecular and Cellular Biology, 24(14):6379-6392, July 2004. ISSN 0270-7306, 1098-5549. doi: 10.1128/MCB.24.14.6379-6392.2004.

77. Haruhiko Asakawa, Hui-Ju Yang, Takaharu G. Yamamoto, Chizuru Ohtsuki, Yuji Chikashige, Kumiko Sakata-Sogawa, Makio Tokunaga, Masaaki Iwamoto, Yasushi Hiraoka, and Tokuko Haraguchi. Characterization of nuclear pore complex components in fission yeast Schizosaccharomyces pombe. Nucleus, 5(2):149-162, March 2014. ISSN 1949-1034. doi: 10.4161/nucl.28487.

78. Maria Makarova, Ying Gu, Jun-Song Chen, Janel Renée Beckley, Kathleen Louise Gould, and Snezhana Oliferenko. Temporal Regulation of Lipin Activity Diverged to Account for Differences in Mitotic Programs. Current Biology, 26(2):237-243, January 2016. ISSN 0960-9822. doi: 10.1016/j.cub.2015.11.061

79. Y. Kim, M. S. Gentry, T. E. Harris, S. E. Wiley, J. C. Lawrence, and J. E. Dixon. A conserved phosphatase cascade that regulates nuclear membrane biogenesis. Proceedings of the National Academy of Sciences, 104(16):6596-6601, April 2007. ISSN 0027-8424, 10916490. doi: 10.1073/pnas.0702099104.

80. S. Siniossoglou, H. Santos-Rosa, J. Rappsilber, M. Mann, and E. Hurt. A novel complex of membrane proteins required for formation of a spherical nucleus. The EMBO journal, 17(22):6449-6464, November 1998. ISSN 0261-4189. doi: 10.1093/emboj/17.22.6449.

81. T. Hirano, G. Konoha, T. Toda, and M. Yanagida. Essential roles of the RNA polymerase I largest subunit and DNA topoisomerases in the formation of fission yeast nucleolus. The Journal of Cell Biology, 108(2):243-253, February 1989. ISSN 0021-9525. doi: 10.1083/ jcb.108.2.243.

82. M. Jiménez, T. Petit, C. Gancedo, and C. Goday. The alm1 + gene from Schizosaccharomyces pombe encodes a coiled-coil protein that associates with the medial region during mitosis. Molecular and General Genetics MGG, 262(6):921-930, January 2000. ISSN 0026-8925, 1432-1874. doi: 10.1007/PL00008660.

83. Jingjing Chen, Jennifer M. Gardner, Zulin Yu, Sarah E. Smith, Sean McKinney, Brian D. 
bioRxiv preprint doi: https://doi.org/10.1101/2021.08.11.455278; this version posted August 11, 2021. The copyright holder for this preprint (which was not certified by peer review) is the author/funder, who has granted bioRxiv a license to display the preprint in perpetuity. It is made available under aCC-BY 4.0 International license.

E Electron microscopy

Slaughter, Jay R. Unruh, and Sue L. Jaspersen. Yeast centrosome components form a noncanonical LINC complex at the nuclear envelope insertion site. J Cell Biol, page jcb.201809045, March 2019. ISSN 0021-9525, 1540-8140. doi: 10.1083/jcb.201809045.

84. Shannon Burns, Jennifer S. Avena, Jay R. Unruh, Zulin Yu, Sarah E. Smith, Brian D. Slaughter, Mark Winey, and Sue L. Jaspersen. Structured illumination with particle averaging reveals novel roles for yeast centrosome components during duplication. eLife, 4 e08586, September 2015. ISSN 2050-084X. doi: 10.7554/eLife.08586.

85. Andrew J. Bestul, Zulin Yu, Jay R. Unruh, and Sue L. Jaspersen. Molecular model of fission yeast centrosome assembly determined by superresolution imaging. J Cell Biol, page jcb.201701041, June 2017. ISSN 0021-9525, 1540-8140. doi: 10.1083/jcb.201701041.

86. Andrew J. Bestul, Zulin Yu, Jay R. Unruh, and Sue L. Jaspersen. Redistribution of centrosomal proteins by centromeres and Polo kinase controls partial nuclear envelope breakdown in fission yeast. Molecular Biology of the Cell, pages mbc.E21-05-0239, June 2021. ISSN 1059-1524, 1939-4586. doi: 10.1091/mbc.E21-05-0239.

87. P. T. Tran, L. Marsh, V. Doye, S. Inoué, and F. Chang. A mechanism for nuclear positioning in fission yeast based on microtubule pushing. The Journal of Cell Biology, 153(2):397411, April 2001. ISSN 0021-9525.

88. Kenneth E Sawin, Paula C. C Lourenco, and Hilary A Snaith. Microtubule Nucleation at Non-Spindle Pole Body Microtubule-Organizing Centers Requires Fission Yeast Centrosomin-Related Protein mod20p. Current Biology, 14(9):763-775, May 2004. ISSN 0960-9822. doi: 10.1016/j.cub.2004.03.042.

89. F. Miki, A. Kurabayashi, Y. Tange, K. Okazaki, M. Shimanuki, and O. Niwa. Two-hybrid search for proteins that interact with Sad1 and Kms1, two membrane-bound components of the spindle pole body in fission yeast. Molecular genetics and genomics: MGG, 270(6): 449-461, January 2004. ISSN 1617-4615. doi: 10.1007/s00438-003-0938-8.

90. Megan C. King, Theodore G. Drivas, and Günter Blobel. A Network of Nuclear Envelope Membrane Proteins Linking Centromeres to Microtubules. Cell, 134(3):427-438, August 2008. ISSN 0092-8674. doi: 10.1016/j.cell.2008.06.022.

91. Paola Gallardo, Ramón R. Barrales, Rafael R. Daga, and Silvia Salas-Pino. Nuclear Mechanics in the Fission Yeast. Cells, 8(10):1285, October 2019. ISSN 2073-4409. doi: $10.3390 /$ cells 8101285

92. Yasushi Hiraoka, Hiromi Maekawa, Haruhiko Asakawa, Yuji Chikashige, Tomoko Kojidani, Hiroko Osakada, Atsushi Matsuda, and Tokuko Haraguchi. Inner nuclear membrane protein Ima1 is dispensable for intranuclear positioning of centromeres: S. pombe inner nuclear membrane proteins. Genes to Cells, 16(10):1000-1011, October 2011. ISSN 13569597. doi: 10.1111/j.1365-2443.2011.01544.x

93. Alfonso Fernández-Álvarez, Cécile Bez, Eileen T. O'Toole, Mary Morphew, and Julia Promisel Cooper. Mitotic Nuclear Envelope Breakdown and Spindle Nucleation Are Controlled by Interphase Contacts between Centromeres and the Nuclear Envelope. Developmental Cell, 39(5):544-559, 2016. ISSN 1878-1551. doi: 10.1016/j.devcel.2016.10. 021.

94. Ramón Ramos Barrales, Marta Forn, Paula Raluca Georgescu, Zsuzsa Sarkadi, and Sigurd Braun. Control of heterochromatin localization and silencing by the nuclear membrane protein Lem2. Genes \& Development, 30(2):133-148, January 2016. ISSN 1549-5477. doi: 10.1101/gad.271288.115.

95. Yanira Gonzalez, Akira Saito, and Shelley Sazer. Fission yeast Lem2 and Man1 perform fundamental functions of the animal cell nuclear lamina. Nucleus (Austin, Tex.), 3(1):6076, February 2012. ISSN 1949-1042. doi: 10.4161/nucl.18824.

96. Susan L. Forsburg. Comparison of Schizosaccharomyces pombe expression systems. Nucleic Acids Research, 21(12):2955-2956, 1993. ISSN 0305-1048, 1362-4962. doi: 10.1093/nar/21.12.2955.

97. Gabriele Basi, Elisabeth Schmid, and Kinsey Maundrell. TATA box mutations in the Schizosaccharomyces pombe nmt1 promoter affect transcription efficiency but not the transcription start point or thiamine repressibility. Gene, 123(1):131-136, January 1993. ISSN 03781119. doi: 10.1016/0378-1119(93)90552-E.

98. H. Funabiki, I. Hagan, S. Uzawa, and M. Yanagida. Cell cycle-dependent specific positioning and clustering of centromeres and telomeres in fission yeast. The Journal of Cell Biology, 121(5):961-976, June 1993. ISSN 0021-9525. doi: 10.1083/jcb.121.5.961.

99. Haitong Hou, Zhou Zhou, Yu Wang, Jiyong Wang, Scott P. Kallgren, Tatiana Kurchuk, Elizabeth A. Miller, Fred Chang, and Songtao Jia. Csi1 links centromeres to the nuclear envelope for centromere clustering. Journal of Cell Biology, 199(5):735-744, November 2012. ISSN 1540-8140, 0021-9525. doi: 10.1083/jcb.201208001.

100. Fan Zheng, Tianpeng Li, Dong-Yan Jin, Viktoriya Syrovatkina, Kathleen Scheffler, Phong T. Tran, and Chuanhai Fu. Csi1p recruits alp7p/TACC to the spindle pole bodies for bipolar spindle formation. Molecular Biology of the Cell, 25(18):2750-2760, September 2014. ISSN 1939-4586. doi: 10.1091/mbc.E14-03-0786.

101. Yoshie Tange, Yuji Chikashige, Shinya Takahata, Kei Kawakami, Masato Higashi, Chie Mori, Tomoko Kojidani, Yasuhiro Hirano, Haruhiko Asakawa, Yota Murakami, Tokuko Haraguchi, and Yasushi Hiraoka. Inner nuclear membrane protein Lem2 augments heterochromatin formation in response to nutritional conditions. Genes to Cells: Devoted to Molecular \& Cellular Mechanisms, 21(8):812-832, August 2016. ISSN 1365-2443. doi: $10.1111 /$ gtc. 12385 .

102. Mingyu Gu, Dollie LaJoie, Opal S. Chen, Alexander von Appen, Mark S. Ladinsky, Michael J. Redd, Linda Nikolova, Pamela J. Bjorkman, Wesley I. Sundquist, Katharine S. Ullman, and Adam Frost. LEM2 recruits CHMP7 for ESCRT-mediated nuclear envelope closure in fission yeast and human cells. Proceedings of the National Academy of Sciences of the United States of America, 114(11):E2166-E2175, March 2017. ISSN 1091-6490. doi: 10.1073/pnas.1613916114

103. Hani Ebrahimi, Hirohisa Masuda, Devanshi Jain, and Julia Promisel Cooper. Distinct 'safe zones' at the nuclear envelope ensure robust replication of heterochromatic chromosome regions. eLife, 7:e32911, May 2018. ISSN 2050-084X. doi: 10.7554/eLife.32911.

104. Kazunori Kume, Helena Cantwell, Alana Burrell, and Paul Nurse. Nuclear membrane protein Lem2 regulates nuclear size through membrane flow. Nature Communications, 10 (1):1871, April 2019. ISSN 2041-1723. doi: 10.1038/s41467-019-09623-x.

105. Birthe Fahrenkrog, Eduard C. Hurt, Ueli Aebi, and Nelly Panté. Molecular Architecture of the Yeast Nuclear Pore Complex: Localization of Nsp1p Subcomplexes. Journal of Cell
Biology, 143(3):577-588, November 1998. ISSN 0021-9525, 1540-8140. doi: 10.1083/jcb. 143.3.577.

106. Viktoriya Syrovatkina, Chuanhai Fu, and Phong T. Tran. Antagonistic spindle motors and MAPs regulate metaphase spindle length and chromosome segregation. Current biology: CB, 23(23):2423-2429, December 2013. ISSN 1879-0445. doi: 10.1016/j.cub.2013.10.023.

107. Gerald Lim H. W., Greg Huber, Yoshihiro Torii, Aiko Hirata, Jonathan Miller, and Shelley Sazer. Vesicle-Like Biomechanics Governs Important Aspects of Nuclear Geometry in Fission Yeast. PLOS ONE, 2(9), September 2007. ISSN 1932-6203. doi: 10.1371/journal. pone.0000948.

108. Evgeny Onischenko, Elad Noor, Jonas S. Fischer, Ludovic Gillet, Matthias Wojtynek, Pascal Vallotton, and Karsten Weis. Maturation Kinetics of a Multiprotein Complex Revealed by Metabolic Labeling. Cell, 183(7):1785-1800.e26, December 2020. ISSN 00928674. doi: 10.1016/j.cell.2020.11.001.

109. Bernhard Hampoelz, Marie-Therese Mackmull, Pedro Machado, Paolo Ronchi, Khanh Huy Bui, Nicole Schieber, Rachel Santarella-Mellwig, Aleksandar Necakov, Amparo AndrésPons, Jean Marc Philippe, Thomas Lecuit, Yannick Schwab, and Martin Beck. Preassembled Nuclear Pores Insert into the Nuclear Envelope during Early Development. Cell, 166(3):664-678, July 2016. ISSN 1097-4172. doi: 10.1016/j.cell.2016.06.015.

110. Bernhard Hampoelz, Andre Schwarz, Paolo Ronchi, Helena Bragulat-Teixidor, Christian Tischer, Imre Gaspar, Anne Ephrussi, Yannick Schwab, and Martin Beck. Nuclear Pores Assemble from Nucleoporin Condensates During Oogenesis. Cell, 179(3):671-686.e17, October 2019. ISSN 1097-4172. doi: 10.1016/j.cell.2019.09.022.

111. T. Toda, M. Shimanuki, and M. Yanagida. Fission yeast genes that confer resistance to staurosporine encode an AP-1-like transcription factor and a protein kinase related to the mammalian ERK1/MAP2 and budding yeast FUS3 and KSS1 kinases. Genes \& Development, 5(1):60-73, January 1991. ISSN 0890-9369. doi: 10.1101/gad.5.1.60.

112. K. Takeshige, M. Baba, S. Tsuboi, T. Noda, and Y. Ohsumi. Autophagy in yeast demonstrated with proteinase-deficient mutants and conditions for its induction. The Journal of Cell Biology, 119(2):301-311, October 1992. ISSN 0021-9525. doi: 10.1083/jcb.119.2.301.

113. M. Baba, K. Takeshige, N. Baba, and Y. Ohsumi. Ultrastructural analysis of the autophagic process in yeast: detection of autophagosomes and their characterization. The Journal of Cell Biology, 124(6):903-913, March 1994. ISSN 0021-9525. doi: 10.1083/jcb.124.6.903.

114. Chia-Wei Lee, Florian Wilfling, Paolo Ronchi, Matteo Allegretti, Shyamal Mosalaganti, Stefan Jentsch, Martin Beck, and Boris Pfander. Selective autophagy degrades nuclear pore complexes. Nature Cell Biology, 22(2):159-166, February 2020. ISSN 1476-4679. doi: 10.1038/s41556-019-0459-2.

115. Javier Fernandez-Martinez, Jeremy Phillips, Matthew D. Sekedat, Ruben Diaz-Avalos, Javier Velazquez-Muriel, Josef D. Franke, Rosemary Williams, David L. Stokes, Brian T. Chait, Andrej Sali, and Michael P. Rout. Structure-function mapping of a heptameric module in the nuclear pore complex. The Journal of Cell Biology, 196(4):419-434, February 2012. ISSN 1540-8140. doi: 10.1083/jcb.201109008.

116. Sarah A. Nordeen, Kasper R. Andersen, Kevin E. Knockenhauer, Jessica R. Ingram, Hidde L. Ploegh, and Thomas U. Schwartz. A nanobody suite for yeast scaffold nucleoporins provides details of the nuclear pore complex structure. Nature Communications, 11(1):6179, December 2020. ISSN 2041-1723. doi: 10.1038/s41467-020-19884-6.

117. Dan Zhang and Snezhana Oliferenko. Tts1, the fission yeast homologue of the TMEM33 family, functions in NE remodeling during mitosis. Molecular Biology of the Cell, 25(19): 2970-2983, October 2014. ISSN 1059-1524, 1939-4586. doi: 10.1091/mbc.E13-12-0729.

118. Bailey A. Koch, Elizabeth Staley, Hui Jin, and Hong-Guo Yu. The ESCRT-III complex is required for nuclear pore complex sequestration and regulates gamete replicative lifespan in budding yeast meiosis. Nucleus (Austin, Tex.), 11(1):219-236, December 2020. ISSN 1949-1042. doi: 10.1080/19491034.2020.1812872.

119. Sue L. Jaspersen and Suman Ghosh. Nuclear envelope insertion of spindle pole bodies and nuclear pore complexes. Nucleus, 3(3):226-236, May 2012. ISSN 1949-1034. doi: 10.4161/nucl.20148.

120. Judite Costa, Chuanhai Fu, V. Mohini Khare, and Phong T. Tran. csi2p modulates microtubule dynamics and organizes the bipolar spindle for chromosome segregation. Molecular Biology of the Cell, 25(24):3900-3908, December 2014. ISSN 1939-4586. doi: 10.1091/mbc.E14-09-1370.

121. Colin P. C. De Souza, Aysha H. Osmani, Shahr B. Hashmi, and Stephen A. Osmani. Partial Nuclear Pore Complex Disassembly during Closed Mitosis in Aspergillus nidulans. Current Biology, 14(22):1973-1984, November 2004. ISSN 0960-9822. doi: 10.1016/j.cub.2004. 10.050 .

122. Paola Gallardo, Paula Real-Calderón, Ignacio Flor-Parra, Silvia Salas-Pino, and Rafael R. Daga. Acute Heat Stress Leads to Reversible Aggregation of Nuclear Proteins into Nucleolar Rings in Fission Yeast. Cell Reports, 33(6):108377, November 2020. ISSN 22111247. doi: 10.1016/j.celrep.2020.108377.

123. Anne C. Meinema, Theo Aspert, Sung Sik Lee, Gilles Charvin, and Yves Barral. Specialization of chromatin-bound nuclear pore complexes promotes yeast aging. July 2021. doi: 10.1101/2021.06.28.450139.

124. Joseph L. Campbell, Alexander Lorenz, Keren L. Witkin, Thomas Hays, Josef Loidl, and Orna Cohen-Fix. Yeast Nuclear Envelope Subdomains with Distinct Abilities to Resist Membrane Expansion. Molecular Biology of the Cell, 17(4):1768-1778, April 2006. ISSN 1059-1524, 1939-4586. doi: 10.1091/mbc.e05-09-0839.

125. J. Bähler, J. Q. Wu, M. S. Longtine, N. G. Shah, A. McKenzie, A. B. Steever, A. Wach, P. Philippsen, and J. R. Pringle. Heterologous modules for efficient and versatile PCRbased gene targeting in Schizosaccharomyces pombe. Yeast (Chichester, England), 14 (10):943-951, July 1998. ISSN 0749-503X. doi: 10.1002/(SICI)1097-0061(199807)14: 10<943::AID-YEA292>3.0.CO;2-Y.

126. Johanne M. Murray, Adam T. Watson, and Antony M. Carr. Transformation of Schizosaccharomyces pombe: Lithium Acetate/ Dimethyl Sulfoxide Procedure. Cold Spring Harbor Protocols, 2016(4):pdb.prot090969, April 2016. ISSN 1940-3402, 1559-6095. doi: 10.1101/pdb.prot090969. Publisher: Cold Spring Harbor Laboratory Press.

127. Janni Petersen and Paul Russell. Growth and the Environment of Schizosaccharomyces pombe. Cold Spring Harbor protocols, 2016(3):pdb.top079764, March 2016. ISSN 19403402. doi: $10.1101 /$ pdb.top079764. 
bioRxiv preprint doi: https://doi.org/10.1101/2021.08.11.455278; this version posted August 11, 2021. The copyright holder for this preprint

(which was not certified by peer review) is the author/funder, who has granted bioRxiv a license to display the preprint in perpetuity. It is made available under aCC-BY 4.0 International license.

128. Aleksandar Vještica, Magdalena Marek, Pedro Junior Nkosi, Laura Merlini, Gaowen Liu, Melvin Bérard, Ingrid Billault-Chaumartin, and Sophie G. Martin. A toolbox of stable integration vectors in the fission yeast Schizosaccharomyces pombe. Journal of Cell Science, 133(1):jcs240754, January 2020. ISSN 1477-9137. doi: 10.1242/jcs.240754.

129. Caroline A. Schneider, Wayne S. Rasband, and Kevin W. Eliceiri. NIH Image to ImageJ: 25 years of image analysis. Nature Methods, 9(7):671-675, July 2012. ISSN 1548-7105. 
A
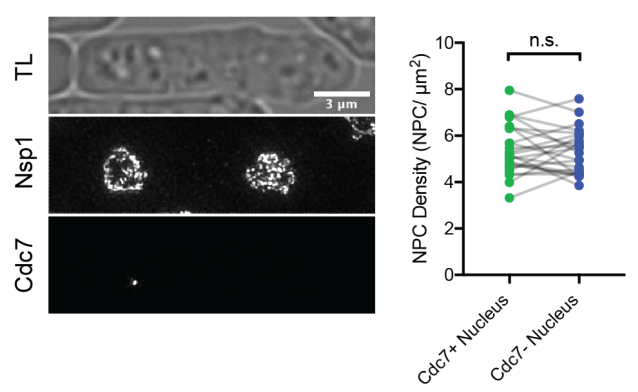

C Simulated 3D-SIM: NPCs/ $/ \mathrm{m}^{2}$
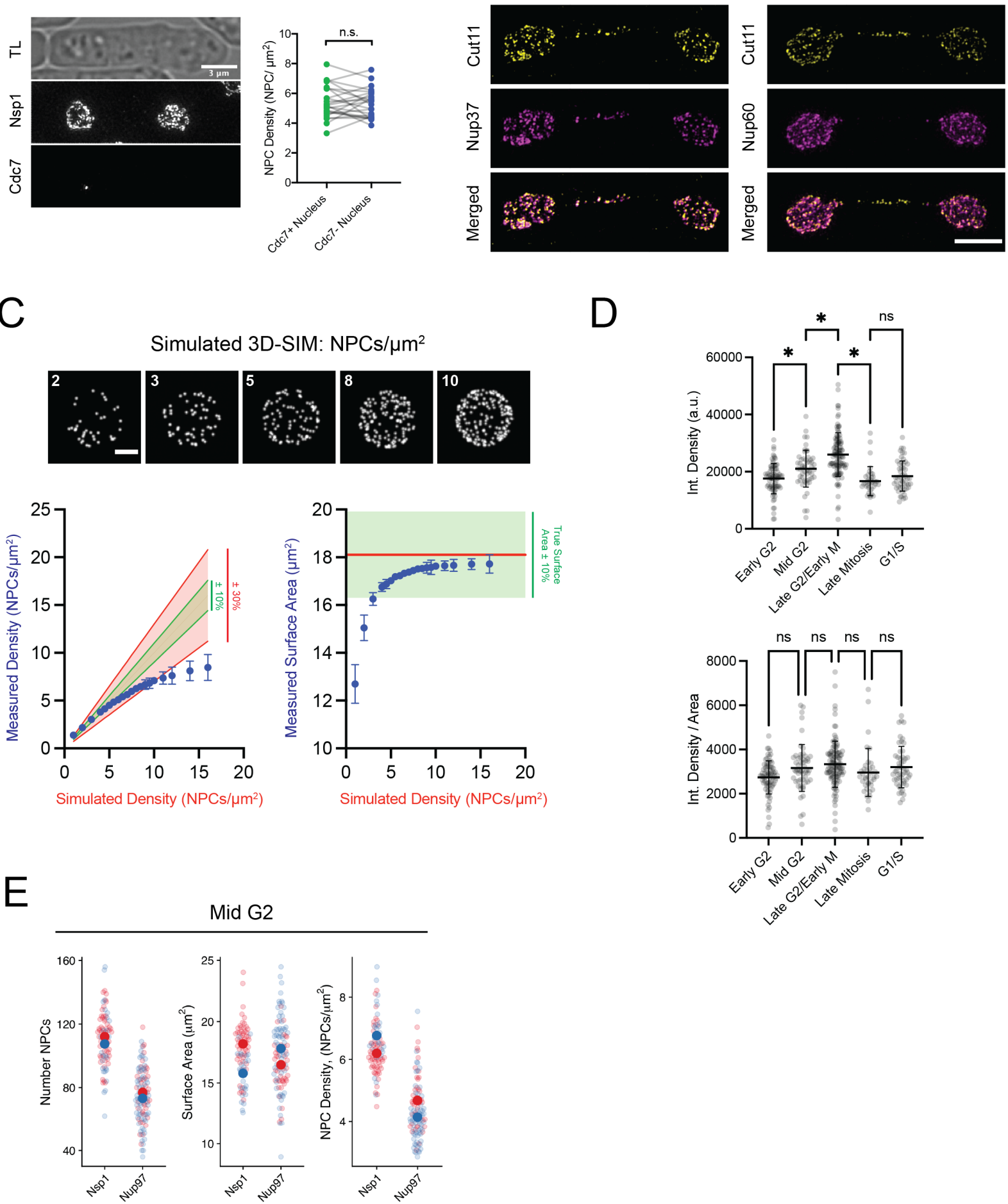

Fig. S1. NPC analysis A) 3D-SIM of Nsp1-mCh NPCs and Cdc7-GFP, which marks the "new" SPB. Quantitation of NPC density in nuclei that did and did not inherit Cdc7-GFP. Bar, $3 \mu \mathrm{m}$. ns, non-significant differences determined by Wilcoxon matched-pairs signed rank test. B) 3D-SIM projections of late anaphase nuclei with Cut11-GFP (yellow) and Nup37-mCh or Nup60-mCh (magenta). Bar, $3 \mu \mathrm{m}$. Loss of Nup60-GFP in the midzone region is due to NPC disassembly (Dey et al, 2020; Expósito-Serrano, et al., 2020). C) Projections of simulated 3D NPC distributions at densities ranging from 2-10 NPCs $/ \mu m^{2}$. Bar, $1 \mu \mathrm{m}$. Graphs comparing the true simulated NPC density and surface areas with the values obtained from the NPC analysis pipeline outlined in Figure 1C. Predicted ranges for values with 10\% (green) and/or 30\% (red) error ranges are highlighted. D) Quantitation of total Nsp1-GFP signal (integrated density) (top), and signal normalized to the nuclear area, validating increases in Nsp1-GFP NPCs through the cell cycle while NPC density is maintained. Asterisk indicates significant difference in mean values determined by One-Way ANOVA with Šidák's multiple comparisons test. E) Comparison of NPC number and densities using Nsp1-GFP and Nup97-GFP. 

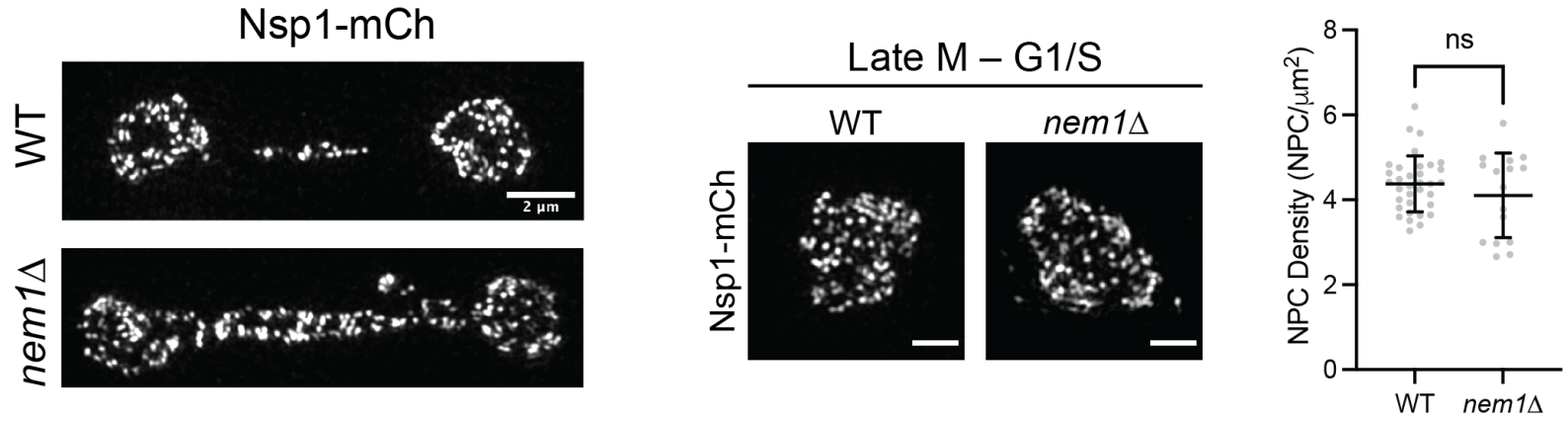

Fig. S2. NPC sequestration to the anaphase bridge in nem1 $\Delta$ D-SIM of Nsp1-mCh NPCs in wild-type and nem1 nuclei in late anaphase. Bar, $2 \mu \mathrm{m}$. nem1 $1 \Delta$ nuclei have normal morphologies and equivalent NPC densities as wild-type nuclei after completing mitosis (Late $\mathrm{M}$ and $\mathrm{G} 1 / \mathrm{S}$, determined by Mann-Whitney test). Bar, $1 \mu \mathrm{m}$.

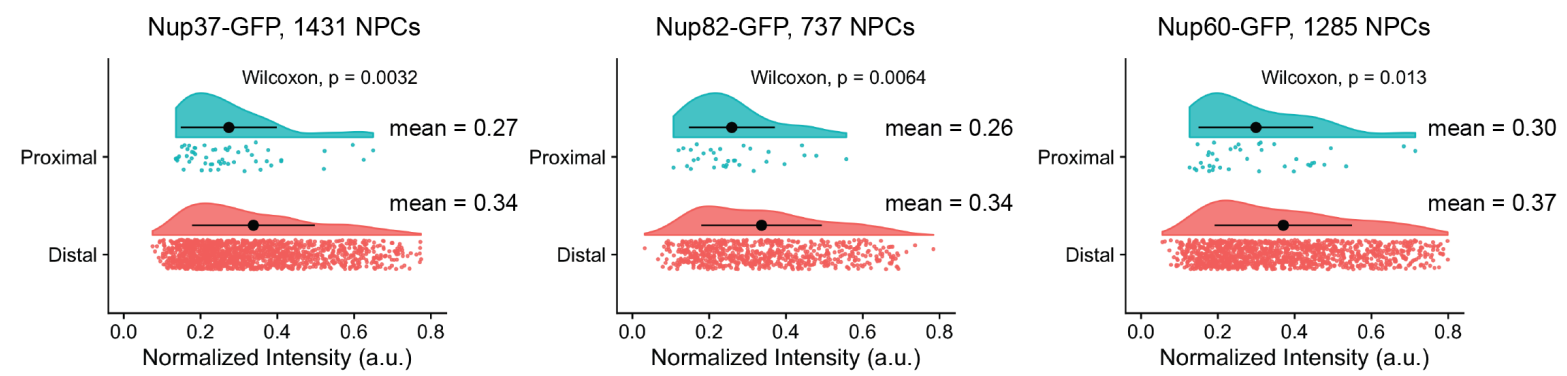

Fig. S3. Nup intensity proximal to the SPB Nup-GFP intensity in SPB-proximal $(<100 \mathrm{~nm})$ and distal $(>100 \mathrm{~nm})$ regions. Values in each were compared using a Wilcoxon rank sum test. Black dots represent the mean normalized intensity value, and error bars show SD.

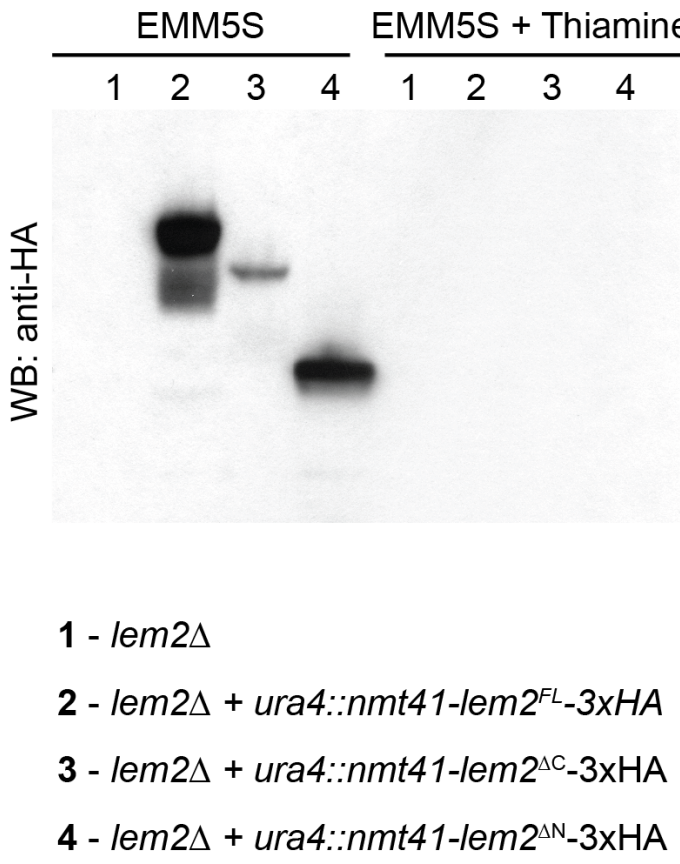

Fig. S4. Expression of Lem2 rescue constructs Immunoblot of strains from Figure 6D. Expression of each construct was observed for each fusion protein by anti-HA immuno blotting in cells cultured in EMM5S (no thiamine) and is reduced to undetectable levels after 22 hours of culture in EMM5S with $15 \mu \mathrm{M}$ thiamine. A Coomassie stained gel is shown as a loading control. The position of molecular weight markers $(\mathrm{kDa})$ is shown. 


\section{Supplemental Tables}

Table S1: Summary statistics for Nsp1-GFP NPC analysis

\begin{tabular}{|c|c|c|c|c|c|c|c|c|}
\hline Stage & $\begin{array}{l}\text { Cell } \\
\text { Length } \\
\text { ( } \mu \mathrm{m})\end{array}$ & $\begin{array}{l}\text { Surface } \\
\text { Area } \\
\left(\mu \mathrm{m}^{2}\right)\end{array}$ & $\begin{array}{l}\text { Volume } \\
\left(\mu \mathrm{m}^{3}\right)\end{array}$ & Sphericity & $\begin{array}{l}\text { Number } \\
\text { NPCs }\end{array}$ & $\begin{array}{l}\text { NPC } \\
\text { Density } \\
\text { (NPCs per } \\
\mu \mathrm{m}^{2} \text { ) }\end{array}$ & $\begin{array}{l}\text { Points } \\
\text { Removed }\end{array}$ & $\mathbf{N}$ \\
\hline Early G2 & $8.7 \pm 0.5$ & $16.9 \pm 2.7$ & $5.9 \pm 1.4$ & $0.93 \pm 0.02$ & $95.3 \pm 19.9$ & $5.7 \pm 1.0$ & $0.8 \pm 1.2$ & 233 \\
\hline Mid G2 & $10.2 \pm 0.4$ & $18.4 \pm 2.8$ & $6.7 \pm 1.6$ & $0.93 \pm 0.02$ & $105.1 \pm 20.2$ & $5.8 \pm 1.0$ & $0.9 \pm 1.5$ & 174 \\
\hline $\begin{array}{l}\text { Late G2/ } \\
\text { Early M }\end{array}$ & $12.7 \pm 1.2$ & $21.8 \pm 4.1$ & $8.7 \pm 2.4$ & $0.93 \pm 0.02$ & $123.7 \pm 26.6$ & $5.8 \pm 1.2$ & $0.9 \pm 1.3$ & 317 \\
\hline Late $\mathrm{M}$ & $14.0 \pm 0.9$ & $14.6 \pm 2.6$ & $4.6 \pm 1.3$ & $0.91 \pm 0.02$ & $77.5 \pm 13.9$ & $5.4 \pm 1.1$ & $1.2 \pm 1.8$ & 122 \\
\hline G1/S & $14.2 \pm 1.0$ & $15.5 \pm 2.8$ & $5.1 \pm 0.5$ & $0.92 \pm 0.02$ & $81.1 \pm 17.1$ & $5.3 \pm 0.9$ & $0.9 \pm 1.2$ & 162 \\
\hline
\end{tabular}

Summary statistics from four independent biological replicates of Nsp1-GFP 3D-SIM imaging experiments. Stages assigned as described in Materials and Methods. Surface Area, Volume, Sphericity and NPC Density values derived from the computed 3D convex hull. Points Removed represents the average number of NPC points removed during complex hull optimization as described in Materials and Methods. 


\section{Table S2: Yeast strains}

\begin{tabular}{|c|c|c|c|}
\hline Strain & Genotype & Figure & Source/Reference \\
\hline fySL707 & $\begin{array}{l}\text { h-, nsp1-GFP-KanMX6, his3-D1, leu1-32, ura4-D18, } \\
\text { ade6-M210 }\end{array}$ & $\begin{array}{l}\text { Fig. 1A-E, } \\
\text { S1D, S1E }\end{array}$ & This study \\
\hline fySL485 & $\begin{array}{l}\text { h-, nup146-GFP-KanMX6, his3-D1, leu1-32, ura4-D18, } \\
\text { ade6-M210 }\end{array}$ & Fig. 1B & This study \\
\hline fySL748 & $\begin{array}{l}\text { h+, nup37-GFP-KanMX6, his3-D1, leu1-32, ura4-D18, } \\
\text { ade6-M210 }\end{array}$ & Fig. 1B & This study \\
\hline fySLJ484 & $\begin{array}{l}\text { h-, nup97-GFP-KanMX6, his3-D1, leu1-32, ura4-D18, } \\
\text { ade6-M210 }\end{array}$ & Fig. 1B, S1E & This study \\
\hline fySL035 & h-, cut11:GFP:ura4+, leu1-32, ura4-D18 & Fig. 1B & $\begin{array}{l}\text { J.R. Mclntosh, } \\
(\mathrm{Mcl} 316)\end{array}$ \\
\hline fySL730 & $\begin{array}{l}\text { h-, nup60-GFP-KanMX6, his3-D1, leu1-32, ura4-D18, } \\
\text { ade6-M210 }\end{array}$ & Fig. 1B & This study \\
\hline fySப714 & $\begin{array}{l}\text { h-, cdc7-GFP-KanMX6, nsp1-mCherry-NatMX6, his3-D1, } \\
\text { leu1-32, ura4-D18, ade6-M210 }\end{array}$ & Fig. S1A & This study \\
\hline fySL771 & $\begin{array}{l}\text { h?, cut11:GFP:ura4t, nup37-mCherry-NatMX6, leu1-32, } \\
\text { ura4-D18 }\end{array}$ & Fig. S1B & This study \\
\hline fySLJ772 & $\begin{array}{l}\text { h?, cut11:GFP:ura4t, nup60-mCherry-NatMX6, leu1-32, } \\
\text { ura4-D18 }\end{array}$ & Fig. S1B & This study \\
\hline fySL4457 & $\begin{array}{l}\text { h+, nsp1-mCherry-NatMX6, his3-D1, leu1-32, ura4-D18, } \\
\text { ade6-M210 }\end{array}$ & Fig. $2 A, 3$ & This study \\
\hline fySL944 & $\begin{array}{l}\text { h+, wee1-50, nup37-mCherry-HygMX6, ade6-M210, } \\
\text { leu1-32, ura4-D18 }\end{array}$ & Fig. 2B & This study \\
\hline fySL1118 & $\begin{array}{l}h ?, \text { ppc89-mCherry-NatMX6, nsp1-GFP-KanMX6, } \\
\text { cdc25.22, leu1-32, ura4-D18 }\end{array}$ & Fig. 2C & This study \\
\hline fySL541 & $h-$, nsp1-mCherry-NatMX6, (prototroph) & Fig. 2D & This study \\
\hline fySL1135 & $\begin{array}{l}\text { h?, atg1 } 1 \triangle: \text { KanMX6, nsp1-mCherry-NatMX6 } \\
\text { (prototroph) }\end{array}$ & Fig. 2D & This study \\
\hline fySL1136 & $\begin{array}{l}\text { h?, atg8 } 8:: K a n M \times 6, \text { nsp1-mCherry-NatMX6 } \\
\text { (prototroph) }\end{array}$ & Fig. 2D & This study \\
\hline fySL515 & $\begin{array}{l}\text { h?, nup1324::KanMX6, nsp1-mCherry-NatMX6, leu1-32, } \\
\text { ura4-D18 }\end{array}$ & Fig. 3A-C & This study \\
\hline fySL879 & $\begin{array}{l}\text { h?, nem1 } 1: \text { KanMX6, nsp1-mCherry-NatMX6, leu1-32, } \\
\text { ura4-D18 }\end{array}$ & Fig. 3D-E, S2 & This study \\
\hline fySL1147 & $\begin{array}{l}\text { h?, nup1324::KanMX6, nsp1-mCherry-NatMX6, Sad1- } \\
\text { GFP-NatMX6 }\end{array}$ & Fig. 3F,G & This study \\
\hline fySL712 & $\begin{array}{l}\text { h?, nuc1-mCherry-NatMX6, nup44-GFP-KanMX6, his3- } \\
\text { D1, leu1-32, ura4-D18, ade6-M210 }\end{array}$ & Fig. 4A-D & This study \\
\hline fySL713 & $\begin{array}{l}\text { h?, nuc1-mCherry-NatMX6, nup146-GFP-KanMX6, his3- } \\
\text { D1, leu1-32, ura4-D18, ade6-M210 }\end{array}$ & Fig. 4B-D & This study \\
\hline fySLI997 & $\begin{array}{l}\text { h?, nuc1-mCherry-NatMX6, nup124-GFP-KanMX6, his3- } \\
\text { D1, leu1-32, ura4-D18, ade6-M210 }\end{array}$ & Fig. 4B-D & This study \\
\hline fySL998 & $\begin{array}{l}\text { h-, nuc1-mCherry-NatMX6, nup211-GFP-KanMX6, his3- } \\
\text { D1, leu1-32, ura4-D18, ade6-M210 }\end{array}$ & Fig. 4B-D & This study \\
\hline fySL1001 & $\begin{array}{l}\text { h-, nuc1-mCherry-NatMX6, nup97-mNeonGreen- } \\
\text { HygMX6, his3-D1, leu1-32, ura4-D18, ade6-M210 }\end{array}$ & Fig. 4B-D & This study \\
\hline fySL1017 & $\begin{array}{l}\text { h?, nuc1-mCherry-NatMX6, nup60-GFP-KanMX6, his3- } \\
\text { D1, leu1-32, ura4-D18, ade6-M210 }\end{array}$ & Fig. 4B-D & This study \\
\hline
\end{tabular}




\begin{tabular}{|c|c|c|c|}
\hline fySL1020 & $\begin{array}{l}\text { h?, nuc1-mCherry-NatMX6, nup61-GFP-KanMX6, his3- } \\
\text { D1, leu1-32, ura4-D18, ade6-M210 }\end{array}$ & Fig. 4B-D & This study \\
\hline fySLI021 & $\begin{array}{l}\text { h?, nuc1-mCherry-NatMX6, alm1-GFP-KanMX6, his3-D1, } \\
\text { leu1-32, ura4-D18, ade6-M210 }\end{array}$ & Fig. 4B-D & This study \\
\hline fySL1022 & $\begin{array}{l}\text { h?, nuc1-mCherry-NatMX6, seh1-GFP-KanMX6, his3-D1, } \\
\text { leu1-32, ura4-D18, ade6-M210 }\end{array}$ & Fig. 4B-D & This study \\
\hline fySLI1208 & $\begin{array}{l}\text { h+, les1-mNeonGreen-HygMX6, ppc89-mCherry- } \\
\text { NatMX6, his3-D1, leu1-32, ura4-D18, ade6-M210 }\end{array}$ & Fig. 5B & This study \\
\hline fySL738 & $\begin{array}{l}\text { h?, nsp1-GFP-KanMX6, ppc89-mCherry-NatMX6, his3- } \\
\text { D1, leu1-32, ura4-D18, ade6-M210 }\end{array}$ & $\begin{array}{l}\text { Fig. 5B-G, } \\
7 B-C\end{array}$ & This study \\
\hline fySL1122 & h+, sad1-GFP-KanMX6, adh15::mCherry-atb2-NatMX6 & Fig. 5C & This study \\
\hline fySLJ745 & $\begin{array}{l}\text { h-, nup60-GFP-KanMX6, ppc89-mCherry-NatMX6, his3- } \\
\text { D1, leu1-32, ura4-D18, ade6-M210 }\end{array}$ & Fig. 5F-G, S3 & This study \\
\hline fySL746 & $\begin{array}{l}\text { h+, nup82-GFP-KanMX6, ppc89-mCherry-NatMX6, his3- } \\
\text { D1, leu1-32, ura4-D18, ade6-M210 }\end{array}$ & Fig. 5F-G, S3 & This study \\
\hline fySL747 & $\begin{array}{l}\text { h+, nup85-GFP-KanMX6, ppc89-mCherry-NatMX6, his3- } \\
\text { D1, leu1-32, ura4-D18, ade6-M210 }\end{array}$ & Fig. 5F-G & This study \\
\hline fySL 822 & $\begin{array}{l}\text { h90, nup37-GFP-KanMX6, ppc89-mCherry-NatMX6, } \\
\text { his3-D1, leu1-32, ura4-D18, ade6-M210 }\end{array}$ & Fig. 5F-G, S3 & This study \\
\hline fySL530 & $\begin{array}{l}\text { h?, nup97-GFP-KanMX6, ppc89-mCherry-NatMX6, his3- } \\
\text { D1, leu1-32, ura4-D18, ade6-M210 }\end{array}$ & Fig. 5F-G & This study \\
\hline fySL867 & $\begin{array}{l}\text { h?, alm1-GFP-KanMX6, ppc89-mCherry-NatMX6, his3- } \\
\text { D1, leu1-32, ura4-D18, ade6-M210 }\end{array}$ & Fig. 5F-G & This study \\
\hline fySL577 & $\begin{array}{l}\text { h+, lem2-GFP-KanMX6, ppc89-mCherry-NatMX6, his3- } \\
\text { D1, leu1-32, ura4-D18, ade6-M210 }\end{array}$ & Fig. $6 \mathrm{~A}$ & This study \\
\hline fySப845 & $\begin{array}{l}\text { h?, nsp1-GFP-KanMX6, ppc89-mCherry-NatMX6, } \\
\text { ima14::HygMX6, leu1-32, ura4-D18 }\end{array}$ & Fig. 6B & This study \\
\hline fySLป849 & $\begin{array}{l}\text { h?, nsp1-GFP-KanMX6, ppc89-mCherry-NatMX6, } \\
\text { lem2 }:: H y g M X 6 \text {, leu1-32, ura4-D18 }\end{array}$ & Fig. 6B & This study \\
\hline fySLJ860 & $\begin{array}{l}\text { h?, nsp1-GFP-KanMX6, ppc89-mCherry-NatMX6, } \\
\text { man1 }:: H y g M X 6 \text {, leu1-32, ura4-D18 }\end{array}$ & Fig. 6B & This study \\
\hline fySL1209 & $\begin{array}{l}\text { h?, nsp1-GFP-KanMX6, ppc89-mCherry-NatMX6, } \\
\text { csi1 ::HygMX6, leu1-32, ura4-D18 }\end{array}$ & Fig. $6 \mathrm{E}$ & This study \\
\hline fySL1042 & $h+$, bleMX6-nmt3X-bqt1-GBP-mCherry:HygMX6 & Fig. 7C & This study \\
\hline fySLI1104 & $\begin{array}{l}\text { h?, bleMX6-nmt3X-bqt1-GBP-mCherry:HygMX6, nsp1- } \\
\text { GFP-KanMX6, ppc89-mCherry-NatMX6 }\end{array}$ & Fig. 7B,C & This study \\
\hline fySL1124 & h-, bleMX6-nmt3X-bqt1-GBP-mCherry:HygMX6, nsp1- & Fig. 7D-E & This study \\
\hline
\end{tabular}

fySL1117 h?, nsp1-GFP-KanMX6, ppc89-mCherry-NatMX6, lem2A::HygMX6, ura4::nmt41-lem2FL-3xHA-ura4+, leu1-32, ura4-D18

fySL1142 h?, nsp1-GFP-KanMX6, ppc89-mCherry-NatMX6, lem2 $2:$ :HygMX6, ura4::nmt41-lem2 $\triangle$ C-3XHA-ura4+, leu1-32, ura4-D18

fySL1144 h?, nsp1-GFP-KanMX6, ppc89-mCherry-NatMX6, lem2 $2:: H y g M X 6$, ura4::nmt41-lem2 $\mathrm{N}$-3xHA-ura4+, leu1-32, ura4-D18

Fig. 6D, S4 This study

Fig. 6D, S4 This study

Fig. 6D, S4 This study 